\title{
Robust Control and Hot Spots in Dynamic Spatially Interconnected Systems
}

\author{
William Brock*and Anastasios Xepapadeas ${ }^{\dagger}$
}

August 15, 2010

\begin{abstract}
This paper develops linear quadratic robust control theory for a class of spatially invariant distributed control systems that appear in areas of economics such as New Economic Geography, management of ecological systems, optimal harvesting of spatially mobile species, and the like. Since this class of problems has an infinite dimensional state and control space it would appear analytically intractable. We show that by Fourier transforming the problem, the solution decomposes into a countable number of finite state space robust control problems each of which can be solved by standard methods. We use this convenient property to characterize hot spots" which are points in the transformed space that correspond to "breakdown" points in conventional finite dimensional robust control, or where instabilities appear or where the value function loses concavity. We apply our methods to a spatial extension of a well known optimal fishing model.
\end{abstract}

Keywords: Distributed parameter systems, robust control, spatial invariance, hot spot, agglomeration.

JEL Classification: C61, C65, Q22

\section{Introduction}

Two issues have attracted considerable interest in economic theory recently. The first is decision making when the decision maker is trying to make good

${ }^{*}$ Department of Economics, University of Wisconsin, 1180 Observatory Drive, Madison, WI, USA, and Beijer Fellow. e-mail: wbrock@ssc.wisc.edu

${ }^{\dagger}$ Athens University of Economics and Business, Department of International and European Economic Studies, Athens, Greece and Beijer Fellow. e-mail: xepapad@aueb.gr 
choices when she regards her model not as the correct one but as an approximation of the correct one, or to put it differently, when the decision maker has concerns about possible misspecifications of the correct model and wants to incorporate these concerns into the decision-making rules (e.g., Salmon 2002; Hansen and Sargent 2001, 2008; Hansen et al. 2006; JET 2006). The second is decision making when the spatial dimension of underlying problem is explicitly taken into account and the decision maker or a regulator seeks to determine spatially dependent rules. In economics, the spatial dimension has been brought into the picture through new economic geography models (e.g., Krugman 1996, Boucekkine et al. 2009, Desmet and Rossi-Hansberg 2009), but also through models of resource management (e.g. Sanchirico and Wilen 1999, Smith et al. 2009, Brock and Xepapadeas 2008, 2010). In fields like biology or automatic control, systems with spatially distributed parameter aspects in the dynamics have been used to study pattern formation on biological agents (e.g., Murray 2003), the control of infinite platoons of vehicles over time (e.g., Bamieh et al. 2002, Curtain et al. 2008), or groundwater management (e.g., Leizarowitz 2008).

The purpose of the present paper is to bring together these two branches of the literature by studying dynamic economic models with explicit spatial dependence when a regulator has concerns about possible misspecifications of the spatiotemporal evolution of the phenomenon. That is, the regulator regards her model as an approximation of the correct spatiotemporal dynamics and seeks spatially dependent regulation that performs well under the approximating model.

The contribution of this unification is that it allows us to study the optimal regulation of spatially interconnected distributed parameter systems when concerns about model misspecification vary across the spatial domain. Concerns about model misspecification, following Hansen et al. (2006) or Hansen and Sargent (2008), means that the regulator distrusts her model and wants good decisions over a cloud of models that surrounds the regulator's approximating or benchmark model, which are difficult to distinguish with finite data sets. The good or robust decisions are obtained by introducing a fictitious 'adversarial agent' which we will refer to as Nature. Nature promotes robust decision rules by forcing the regulator, who seeks to maximize an objective, to explore the fragility of decision rules to departures from the benchmark model. A robust decision rule to model misspecifica- 
tion means that lower bounds to the rule's performance are determined by Nature - the adversarial agent - who acts as a minimizing agent when constructing these lower bounds. Hansen et al. (2006) show that robust control theory can be interpreted as a recursive version of max-min expected utility theory (Gilboa and Schmeidler 1989). In this context the decision maker cannot or does not formulate a single probability model and maximizes expected utility assuming the probability weights are chosen by Nature, the adversarial agent.

When robust control theory is combined with distributed parameter models, it provides a method for studying robust regulation when the cloud of models surrounding the benchmark model differs among spatial locations. Thus the regulator can design the decision rules not only with respect to the spatial characteristics of the problem but also with respect to the degree to which the regulator distrusts her model across locations. This means that if concerns about the benchmark model in a given site deviate from concerns in other sites, a spatially dependent robust rule should capture these differences. This observation allows us to formally identify, for the first time to our knowledge in economics, spatial hot spots - which are sites where robust control breaks down - or sites where robust control is very costly as a function of the degree of the regulator's concern about model misspecification. We are also able to identify spatial hot spots where the need to apply robust control induces spatial agglomerations and breaks down spatial symmetry. From the theory point of view this is, as far as we know, a new source for generating spatial patterns as compared to the classic Turing diffusion induced instability (Turing 1952) and the more recently identified optimal diffusion or spatial-spillover-induced instabilities (Brock and Xepapadeas, 2008, 2009, 2010).

This unification brings up another point which could be associated with applied policy design and regulation. It has been argued recently (e.g., Haldane 2009) that increased interconnectedness among networks has made various networks, such as ecological networks, power grid networks, transportation networks, financial networks more unstable. This interconnectedness and the instabilities generated at hot spots are captured in our model by the distributed parameter aspect. ${ }^{1}$

\footnotetext{
${ }^{1}$ Although we choose to interpret the characteristics associated with the distributed parameter aspect as physical space, the notion of "space" does not have to be physical.
} 
Distributed parameter models result in optimal control problems in infinite dimensional spaces. By using Fourier methods and exploiting the property of spatial invariance of a class of linear quadratic problems, we are able to obtain solutions to infinite dimensional problems, by solving parameterized finite-dimensional problems. Furthermore, by showing how to obtain correct linear quadratic approximations - in the sense of Magill (1977a,b) and Benigno and Woodford (2006) - of nonlinear distributed parameter robust control problems, we obtain solutions of infinite dimensional robust control problems in terms of linear quadratic approximations of parameterized families of finite dimensional problems. We consider this to be another contribution of this paper.

In sections 2 and 3 we present our theory and we define hot spots. In section 4 we apply our theory to a classic model of commercial fishing (Smith 1969) where spatial interconnections in economic and biological variables are captured by local and non-local spatial effects. We show how a regulator could design optimal spatiotemporal robust control for this fishery, how hot spots emerge, and what implications they might have for regulation.

\section{Robust control in stochastic distributed para- meter systems}

We consider a distributed parameter control system where the state and the control functions are respectively represented by real functions $x(t, z)$ and $u(t, z)$ of time $t \in[0, \infty):=\mathcal{T}$, and a variable $z \in Z$, where $Z$ is a domain that describes a dimension different from the temporal dimension, along which the state and the control functions evolve. Thus $Z$ could be interpreted as a spatial domain, implying that we study spatiotemporal evolutions, or a domain defining social characteristics or describing varieties of goods or sectors of the economy. Technically, and in order to develop a general framework of analysis, $Z$ is a locally compact Abelian group (see for example Rudin (1962) for definitions). Special cases of $Z$ include the real line $\mathbb{R}$, the unit circle $\partial D$, the integers $\mathbb{Z}$, or the finite group of integers modulo $N, \mathbb{Z}_{N}$. For the rest of our analysis we will assume that $Z$ is the finite

It can be used to model characteristics that are associated with economic, sociological, cultural or other factors. Since the notion of "space" may be broadly interpreted, this suggests that our methods can be used for the analysis of a wide range of problems. 
group of integers modulo $N$. This means that our group of characteristics, whether spatial, social, or economic, can be represented by a discrete ring of cells with the property that 'cell 0' is the same as 'cell $N$ ', 'cell 1' is the same as 'cell $N+1$ ' and so on. ${ }^{2}$

Our state and control functions can be identified with the abstract functions $x(t)(z)=x(t, \cdot), u(t)(z)=u(t, \cdot)$, which take values on $Z$ and which belong to the space of vector valued functions which are square integrable with respect to the Haar measure: ${ }^{3},{ }^{4}$

$$
L_{2}^{n}(Z):=\left\{f:\left.Z \rightarrow \Re^{n}\left|\|f\|_{2}^{2}=\int_{Z}\right| f(z)\right|^{2} d z<\infty\right\} .
$$

We introduce into our system the real function $v(t, z)$ which is also identified with the abstract function $v(t)(z)=v(t, \cdot)$ that takes values in the space of functions which are square integrable with respect to the Haar measure This function describes a deterministic specification error which is expressed in terms of deviations from a baseline or benchmark case which is defined for $v(t, z):=0$. This specification error is distributed across the domain $Z$ according to $v(t)(z)$, so that the error may vary across cells at the same point in time. To express the idea that when the model is misspecified the benchmark model remains a good approximation, we restrain the misspecification errors for problems by

$$
\sum_{z=1}^{N}\left[\int_{0}^{\infty} e^{-\rho t}[v(t, z)]^{2} d t\right] \leq v_{0}
$$

where $e^{-\rho t}$ is the appropriate discount factor.

Each cell $z \in Z$ of the system is also subject to a stochastic force which

\footnotetext{
${ }^{2}$ This assumption simplifies considerably the technical aspects of our analysis without any loss in the generality of results, since our analysis can be generalized to continuous spaces. The assumption of 'ring of cells' was used by Turing (1952) in the classic paper on morphogenesis.

${ }^{3}$ The Haar measure is a variant of the Lebesgue measure suitable for $\mathbb{Z}_{N}$. The Haar measure is invariant to the translation map $z \mapsto z+z_{0}$ and the translation operation for functions on $Z$ defined as $\left(T_{z_{0}} f\right)(z):=f\left(z-z_{0}\right)$. An operator $A$ with domain $\mathcal{D}(A)$ in the space of square integrable functions with respect to the Haar measure is said to be translation invariant if for all $z \in Z$

$T_{z}: \mathcal{D}(A) \rightarrow \mathcal{D}(A)$ and $A T_{z} f=T_{z} A f \quad \forall f \in \mathcal{D}(A)$ (Bamieh et al. 2003, p. 1023). As we will see later, translation invariance is very useful for providing tractability to the models developed in this paper, without introducing unrealistic characteristics.

${ }^{4}$ When we write the integral with respect to $d z$ we interpret it as a sum over $z \in \mathbb{Z}_{N}$. In the rest of the paper we use integral signs and sums interchangeably.
} 
is represented by a white noise $\dot{\mathcal{B}}(t)(z)$ which is the formal time-derivative of a Wiener process $\mathcal{B}(t)(z)$ which is placed in each cell $z$.

Thus in our model the coordinates of the characteristics (spatial, social, or economics) denoted by $z$ vary in the group $Z$, and the functions related to state, control and misspecification functions as well as the stochastic force are fully distributed over this coordinate.

Effects across the $z$ coordinate, for example spatial effects, on the state of the system are modelled in terms of local and long-range or nonlocal effects. Nonlocal effects describing the impact of the concentration of the state variable $x\left(t, z^{\prime}\right)$ in cell $z^{\prime}$ on $x(t, z)$ are modelled using the kernel formulation:

$$
(\mathbf{K} x)(t, z):=\sum_{z^{\prime} \in Z} K_{x}\left(z-z^{\prime}\right) x\left(t, z^{\prime}\right)=X(t, z) .
$$

Local effects are modeled by classic diffusion. Interpreting partial derivatives with respect to $z$ as finite differences when working on $\mathbb{Z}_{N}$, local effects are represented by the term

$$
E_{d}(t, z)=D[x(t, z+1)-2 x(t, z)+x(t, z-1)],
$$

where $D>0$ is the diffusion coefficient. ${ }^{5}$

When effects are non-local the degree of interconnectedness can be represented by fixed parameters. For the kernel specification this can be modelled by writing:

$$
\sum_{z^{\prime} \in Z} K_{x}\left(z-z^{\prime}\right) x\left(t, z^{\prime}\right) d z^{\prime}=\alpha_{1} \sum_{z^{\prime} \in Z} e^{-\alpha_{2}\left|z-z^{\prime}\right|} x\left(t, z^{\prime}\right) d z^{\prime}
$$

where $\alpha_{1}$ and $\alpha_{2}$ are level and shape parameters. For example, as $\alpha_{2}$ decreases, the kernel increases and becomes "flatter" at the same time, suggesting that interconnectedness increases. For local effects, interconnectedness is related to the diffusion coefficient $D$. The higher $D$ is, the faster the state variable moves from cells of high concentration to cells of low concentration.

The interconnectedness in our system is also reflected in nonlocal control

\footnotetext{
${ }^{5}$ When $Z$ is continuous then local effects are modeled by $E_{c}(t, z)=D \frac{\partial^{2} x(t, z)}{\partial z^{2}}$.
} 
effects which are modelled, using the kernel formulation, by:

$$
(\mathbf{K} u)(t, z):=\sum_{z^{\prime} \in Z} K_{u}\left(z-z^{\prime}\right) u\left(t, z^{\prime}\right)=U(t, z) .
$$

These effects describe the impact of the control applied in cell $z^{\prime}$ on the control of the system $u(t, z)$ in cell $z$.

The stochastic shocks in each cell can also be correlated across cells. In this case the stochastic term can be defined as

$$
(\mathbf{K} d \mathcal{B})(t, z):=\sum_{z^{\prime} \in Z} K_{\varepsilon}\left(z-z^{\prime}\right) d \mathcal{B}\left(t, z^{\prime}\right)=\varepsilon(t, z)
$$

All the kernel functions, such $K_{j}(\cdot), j=x, u$, used in this paper are assumed to be continuous and symmetric around zero in $Z$, or $K_{j}(z)=$ $K_{j}(-z)$. Given the above assumptions, the evolution of the system's state can described in continuous time domain by by a general equation of motion:

$$
\begin{aligned}
\frac{\partial x(t, z)}{\partial t}= & f(x(t, z), u(t, z),(\mathbf{K} x)(t, z),(\mathbf{K} u)(t, z), v(t, z)) \\
& +E_{d}(t, z)+(\mathbf{K} d \mathcal{B})(t, z), x(0, z)=x_{0}(z) .
\end{aligned}
$$

In a discrete time domain $t=0,1,2, \ldots$, the evolution of the system is described as

$$
\begin{aligned}
x_{t+1}(z)= & f\left(x_{t}(z), u_{t}(z), X_{t}(z), U_{t}(z), v_{t+1}(z)\right) \\
& +E_{d_{t}}(z)+\varepsilon_{t+1}(z), z=1, \ldots, N .
\end{aligned}
$$

The state dynamics (8) or (9) coupled with a payoff functional can be used to extend the robust control analytical framework developed by Hansen and Sargent (2008) to distributed parameter systems. When the regulator has concerns about model misspecification, the regulator's objective can be expressed in terms of the following penalty distributed parameter robust control problem for given $\theta \in(\underline{\theta},+\infty]$ :

$$
\begin{gathered}
\sup _{u(t, z)} \inf _{v(t, z)} E_{0} \sum_{z \in Z}\left\{\int _ { 0 } ^ { \infty } e ^ { - \rho t } \left[f_{0}\left(x(t, z), u(t, z), X_{0}(t, z), U_{0}(t, z)\right)\right.\right. \\
\left.\left.+\theta v^{2}(t, z)\right] d t\right\} \text { subject to }(8)
\end{gathered}
$$


where

$$
\begin{aligned}
& X_{0}(t, z):=\sum_{z^{\prime} \in Z} K_{x}^{0}\left(z-z^{\prime}\right) x\left(t, z^{\prime}\right) d z^{\prime}=\left(\mathbf{K}_{0} x\right)(t, z) \\
& U_{0}(t, z):=\sum_{z^{\prime} \in Z} K_{u}^{0}\left(z-z^{\prime}\right) u\left(t, z^{\prime}\right) d z^{\prime}=\left(\mathbf{K}_{0} u\right)(t, z)
\end{aligned}
$$

represent nonlocal effects in the payoff functional which are modelled using the kernel formulation. In the extremization problem $^{6}(10)$, the minimizing agent - Nature - chooses a $v$ while $\theta \in(\underline{\theta},+\infty], \underline{\theta}>0$ is a penalty parameter restraining the minimizing choice of the $v(t, z)$ function. The lower bound $\underline{\theta}$ is a so-called breakdown point beyond which it is fruitless to seek more robustness because the minimizing agent is sufficiently unconstrained so that she/he can push the criterion function to $-\infty$ despite the best response of the maximizing agent. Thus when $\theta<\underline{\theta}$, robust control rules cannot be attained. The benchmark distributed parameter optimal control problem is a special case of $(10)$ for $v(t, z) \equiv 0$, while when $(\mathbf{K} d \mathcal{B})(t, z) \equiv 0$ in addition, we have a deterministic distributed parameter control problem.

Problem (10) can be regarded as a starting point for defining a robustdistributed parameter linear quadratic regulator problem. This problem, which as far as we know has not been studied before in economics, can be used to provide new insights into the regulation of various applied problems when the regulator has concerns about model misspecification, the state function evolves in time and space and local and nonlocal spatial effects are present.

A special case of an optimal solution for problem (10), provided it exists, is the optimal solution of the spatially independent deterministic benchmark problem. This problem is defined for $v(t, z) \equiv 0, D=0$, $x(t, z)=x(t), u(t, z)=u(t)$. Spatial independence means that the kernel operators can be written as $(\boldsymbol{\Phi} \phi)(t, z)=\phi(t) \sum_{z^{\prime} \in Z} \varphi\left(z-z^{\prime}\right)$, where $\sum_{z^{\prime} \in Z} \varphi\left(z-z^{\prime}\right)=\bar{\varphi}$ is a fixed parameter for given $\varphi(\cdot)$, and $\phi=(x, u), \varphi=$ $\left(\mathbf{K}_{x}^{0}, \mathbf{K}_{x}, \mathbf{K}_{u}^{0}, \mathbf{K}_{u}\right)$. We shall call a locally optimal steady state of the spatially independent deterministic benchmark problem denoted by $\left(\bar{x}^{*}, \bar{u}^{*}, 0\right)$ a flat optimal steady state (FOSS) since this steady state will exhibit a spatially uniform distribution for the state-costate and control variables associated with the problem. In Appendix 1 we show that the correct linear

\footnotetext{
${ }^{6}$ We follow Hansen and Sargent in using the term extremization for the sup inf operation.
} 
quadratic approximation of the robust distributed parameter problem (10) with deterministic misspecification can be written, dropping $(t, z)$ to ease notation, as:

$$
\begin{aligned}
& \sup _{u(t, z)} \inf _{v(t, z)} \sum_{z \in Z}\left[\int_{0}^{\infty} e^{-\rho t} \frac{1}{2}\left[\xi^{\prime} Q \xi+\theta v^{2}(t, z)\right]\right] d t, \\
& \text { subject to } \\
\frac{\partial x}{\partial t}= & A x+B u+\bar{A} X+\bar{B} U+C v+E_{d} \\
& x(0, z)=x_{0}(z), x(t, 0)=x(t . N), \forall t \\
\xi= & \left(x, u, X_{0}, U_{0}, X, U\right),(A, B, \bar{A}, \bar{B}, C): \text { fixed parameters }
\end{aligned}
$$

where, by a slight abuse of notation, $(x, u, v)$ denote deviations from the deterministic FOSS. In (13) $Q=\left[q_{i j}\right], i, j=1, \ldots, 6$ is a $(6 \times 6)$ symmetric matrix of the second derivatives, in the Fréchet sense, of the Hamiltonian of the spatially independent deterministic benchmark problem evaluated at the FOSS and $(A, B, \bar{A}, \bar{B}, C)=\left(f_{x}^{*}, f_{u}^{*}, f_{X}^{*}, f_{U}^{*}, f_{v}^{*}\right)$ with all Fréchet derivatives evaluated at the FOSS (see Appendix 1 for details).

In discrete time the problem can be written as:

$$
\begin{aligned}
& \sup _{u_{t}(z)} \inf _{v_{t}(z)} \sum_{z \in Z} \sum_{t=0}^{\infty} \beta^{t} \frac{1}{2}\left[\xi^{\prime} Q \xi+\theta v^{2}(t, z)\right] \\
& \text { subject to } \\
x_{t+1}= & A x_{t}+B u_{t}+\bar{A} X_{t}+\bar{B} U_{t}+C v_{t+1}+E_{d t} .
\end{aligned}
$$

Problem (13) is a linear quadratic problem. We can think of this problem as the problem of a linear quadratic regulator, or as a linear quadratic approximation of a more general nonlinear penalty distributed parameter robust control problem. ${ }^{7}$

\footnotetext{
${ }^{7}$ Note that the expectation operator is missing from the linear quadratic approximations (13) or (17). This is because, as we explain in detail in section 3, a certainty equivalence principle holds which is related to the Hansen and Sargent result (2008, Section 2.4.1). This principle states that the controls are the same for the deterministic and the stochastic version of the linear quadratic approximation, or equivalently the controls are the same whether or not the stochastic term is included in (14) or (18). In our paper this principle is slightly different from the one of Hansen and Sargent because we don't multiply the shocks, if they were to be included in (14) or (18), by the same matrix $C$ that multiplies the adversarial agent's control, $v(t)$ or $v_{t+1}$.
} 


\section{Robust linear-quadratic regulation and hot spots}

Problem (13) is defined in the infinite dimensional space of functions which are square integrable with respect to the Haar measure. The analysis of this problem can be greatly simplified by exploiting the property of the objective functional and the dynamics of problem (13) to be translation invariant with respect to the coordinate $z$. This property allows us to decompose, using Fourier transforms, the infinite dimensional optimal control problem to a set of finite dimensional optimal control problems (Bamieh et al. 2002). The Fourier transform $\mathcal{F}$ associates a function $\psi(\cdot)$ on the set $Z$ with a function $\hat{\psi}(\omega)$ on the set $\hat{Z}$ which is called the dual or the character group. In our case the discrete Fourier transform (DFT) (e.g., Chu 2008) of a function $\psi(t)(z)=\psi(t, \cdot)=\psi(t, z), z \in Z$ is defined as:

$$
\mathcal{F} \psi(t)(\omega)=\hat{\psi}(t, \omega):=\frac{1}{N} \sum_{z=1}^{N} \psi(t, z) e^{-2 \pi i \omega \frac{z}{N}}, \omega \in \hat{Z} .
$$

When $Z=\mathbb{Z}_{N}$ then $\hat{Z}=\mathbb{Z}_{N}$ as well, thus the Fourier transform $\mathcal{F}$ will map functions on $\mathbb{Z}_{N}$ to functions also on $\mathbb{Z}_{N}$. The inverse Fourier transform is

$$
\psi(t, z):=\sum_{\omega=0}^{N-1} \hat{\psi}(t, \omega) e^{2 \pi i \omega \frac{z}{N}}, z \in \mathbb{Z}_{N}
$$

The properties of the Fourier transform imply that translation invariant operators in $Z$ are associated with multiplication operators in $\hat{Z}$. Since our kernel operators are translation invariant we have, using the convolution theorem for the DFT:

$$
\begin{aligned}
\mathcal{F}\left(K_{j} \phi\right)(t, \omega) & =\left(\widehat{K_{j} \phi}\right)(t, \omega)=\hat{K}_{j}(\omega) \hat{\phi}(t, \omega) \\
j & =x, u, \varepsilon, \phi=(x, u, d \mathcal{B}) .
\end{aligned}
$$

Thus the use of DFT allows us to transform nonlocal effects (spatial or economic) expressed by kernels into simpler multiplicative expressions in $\mathbb{Z}_{N}$. Local effects can also be simplified by using the shift theorem of the DFT, which implies that:

$$
\mathcal{F} x(t)(\omega-k)=e^{-2 \pi i k \frac{\omega}{N}} \hat{x}(t, \omega) .
$$


Using the shift theorem the DFT of the local effect term becomes:

$$
\begin{aligned}
& \mathcal{F} D[x(t, \omega+1)-2 x(t, \omega)+x(t, \omega-1)]= \\
& D\left[\exp \left(\frac{2 \pi i \omega}{N}\right)-2+\exp \left(\frac{-2 \pi i \omega}{N}\right)\right] \hat{x}(t, \omega)= \\
& 2 D\left[\cos \left(\frac{2 \pi \omega}{N}\right)-1\right] \hat{x}(t, \omega)=-4 D \sin ^{2}\left(\frac{\pi \omega}{N}\right) \hat{x}(t, \omega)
\end{aligned}
$$

using in the second and third line the trigonometric identities

$$
\begin{aligned}
\exp (i \theta) & =\cos (\theta)+i \sin (\theta), \exp (-i \theta)=\cos (\theta)-i \sin (\theta) \\
2 \sin ^{2}(\theta) & =1-\cos (2 \theta) .
\end{aligned}
$$

Taking the DFT of the linearization of $(8)$, we obtain: ${ }^{8}$

$$
\begin{aligned}
\frac{d \hat{x}(t, \omega)}{d t}= & A \hat{x}(t, \omega)+B \hat{u}(t, \omega)+\bar{A} \hat{K}_{x}(\omega) \hat{x}(t, \omega)+\bar{B} \hat{K}_{u} \hat{u}(t, \omega) \\
& +C \hat{v}(t, \omega)-4 D \sin ^{2}\left(\frac{\pi \omega}{N}\right) \hat{x}(t, \omega)+\hat{K}_{\varepsilon}(\omega) d \hat{\mathcal{B}}(t, \omega) \text { or } \\
\frac{d \hat{x}(t, \omega)}{d t}= & {\left[A-4 D \sin ^{2}\left(\frac{\pi \omega}{N}\right)+\bar{A} \hat{K}_{x}(\omega)\right] \hat{x}(t, \omega)+\left(B+\bar{B} \hat{K}_{u}(\omega)\right) \hat{u}(t, \omega) } \\
& +C \hat{v}(t, \omega)+\hat{K}_{\varepsilon}(\omega) d \hat{\mathcal{B}}(t, \omega), \hat{x}(0, \omega)=\hat{x}_{0}(\omega) .
\end{aligned}
$$

The Plancherel theorem implies that the quadratic objective functional (13) can be written as

$$
\begin{aligned}
\sup _{u(t, z)} \inf _{v(t, z)} E_{0} \int_{0}^{\infty} e^{-\rho t} \sum_{z \in \mathbb{Z}_{N}}\left[\frac{1}{2}\left[\xi^{\prime} Q \xi+\theta v^{2}(t, z)\right]\right] d t= \\
\sup _{\hat{u}(t, z)} \inf _{\hat{v}(t, z)} \sum_{\omega \in \mathbb{Z}_{N}}\left[\int_{0}^{\infty} e^{-\rho t} \frac{1}{2}\left[\hat{w}^{\prime} \hat{Q} \hat{w}+\theta \hat{v}^{2}(t, \omega)\right] d t\right] \\
\hat{w}^{\prime}=(\hat{x}(t, \omega), \hat{u}(t, \omega))^{\prime}, \hat{Q}=\left[\hat{q}_{i j}(\omega)\right] \quad i, j=1,2,
\end{aligned}
$$

where the elements of matrix $\hat{Q}$ are defined in the dual space $\hat{Z}$, or the frequency domain, and depend on the spatial kernels. In Appendix 2 we show how to derive the quadratic form $\hat{w}^{\prime} \hat{Q} \hat{w}$. Then the linear quadratic approximation of robust distributed parameter problem (10) is equivalent to the extremization of (31) subject to (29). In the same way the discrete

\footnotetext{
${ }^{8}$ We assume that the Wiener processes are the same at equidistant points around the circle so that the output of the Fourier transform is real.
} 
time problem (17) can be written as:

$$
\begin{aligned}
& \sup _{\hat{u}(t, z)} \inf _{\hat{v}(t, z)} E_{0} \sum_{\omega \in \mathbb{Z}_{N}}\left[\sum_{t=0}^{\infty} \beta^{t} \frac{1}{2}\left[\hat{w}^{\prime} \hat{Q} \hat{w}+\beta \hat{v}_{t+1}(\omega)^{2}\right]\right] d \omega \\
& \hat{w}^{\prime}=\left(\hat{x}_{t}(\omega), \hat{u}_{t}(\omega)\right)^{\prime}, \hat{Q}=\left[\hat{q}_{i j}(\omega)\right] \quad i, j=1,2 \\
& \hat{x}_{t+1}(\omega)=\left[A-4 D \sin ^{2}\left(\frac{\pi \omega}{N}\right)+\bar{A} \hat{K}_{x}(\omega)\right] \hat{x}_{t}(\omega)+ \\
& \left(B+\bar{B} \hat{K}_{u}(\omega)\right) \hat{u}_{t}(\omega)+C \hat{v}_{t+1}(\omega)+\hat{K}_{\varepsilon}(\omega) \hat{\varepsilon}_{t+1}(\omega) .
\end{aligned}
$$

The robust linear quadratic regulator problem (31) and (29) with initial conditions $\hat{x}(0, \omega)=\hat{x}_{0}(\omega)$ is "block-diagonal" with blocks parametrized by $\omega$. That is, for a fixed $\omega$ problem (31) and (29) is a finite dimensional linearquadratic penalty robust control problem of the type studied by Hansen and Sargent (2008). We can use problem (31) and (29) or the equivalent problem (33)-(35) to characterize the emergence of a spatial hot spot. We use the continuous time model to characterize hot spots, but results can easily be extended to the discrete time model. Under appropriate regularity assumptions (Hansen et al. 2006), the $\{$ sup, inf $\}$ operators can be replaced with $\{\max , \min \}$. Furthermore the order in which the maximizing agent and the minimizing agent choose does not matter (Hansen and Sargent 2008, Chapter 7, Section 7.7).

Recalling that Fourier transformation diagonalizes the coupled matrix Bellman equation in $z$-space into $N$ separate scalar Bellman equations, one for each $\omega \in \hat{\mathbb{Z}}_{N}$, and suppressing $\omega$ to simplify notation, the Bellman-Isaacs equation for the linear quadratic problem (31) and (29) can be written as the scalar equation below:

$$
\begin{aligned}
-\rho P \hat{x}^{2}-\rho p= & \max _{\hat{u}} \min _{\hat{v}}\left[\frac{1}{2}\left(M \hat{x}^{2}+N \hat{u}^{2}+2 S \hat{x} \hat{u}+\theta \hat{v}^{2}\right)+\right. \\
& \left.(-2 P \hat{x})(F \hat{x}+G \hat{u}+C \hat{v})+\frac{1}{2}(-2 P) K_{\varepsilon}^{2}\right] \\
& M(\omega)=\hat{q}_{11}(\omega), N(\omega)=\hat{q}_{22}(\omega), \quad S(\omega)=\hat{q}_{12}(\omega) \\
& M<0, N<0, M N-S^{2}>0, \forall \omega \text { for strict concavity } \\
& F=A-4 D \sin ^{2}\left(\frac{\pi \omega}{N}\right)+\bar{A}_{x}(\omega), G=B+\bar{B} \hat{K}_{u}(\omega)
\end{aligned}
$$

where $-P \hat{x}^{2}(\omega)-p=V(\hat{x}(\omega))$ is the value function for the problem with $P, p$ parameters to be determined. Following standard approaches we solve 
for the minimization problem first to obtain

$$
\hat{v}(\omega)=\frac{2 P C}{\theta} \hat{x}(\omega)
$$

Substituting into the Bellman-Isaacs equation, the maximization problem is

$$
\begin{aligned}
-\rho P \hat{x}^{2}-\rho p= & \max _{\hat{u}}\left[\frac{1}{2}\left(M \hat{x}^{2}+N \hat{u}^{2}+2 S \hat{x} \hat{u}+\frac{4 P^{2} C^{2}}{\theta} \hat{x}^{2}\right)+\right. \\
& \left.(-2 P \hat{x})\left(F \hat{x}+G \hat{u}+\frac{2 P C^{2}}{\theta} \hat{x}\right)-P K_{\varepsilon}^{2}\right],
\end{aligned}
$$

which implies that the optimal decision rule for the maximizing agent is

$$
\hat{u}(\omega)=\frac{2 P G-S}{N} \hat{x}(\omega) .
$$

Substituting into (39) and equating factors of like power, we obtain that $P$ is determined by the solution of the quadratic expression

$$
\begin{aligned}
& \phi(P)=2\left(\frac{C^{2}}{\theta}+\frac{G^{2}}{N}\right) P^{2}+ \\
& \left(2 F-\rho-\frac{2 G S}{N}\right) P+\left(\frac{S^{2}-M N}{2 N}\right)=0 \\
& F=A-4 D \sin ^{2}\left(\frac{\pi \omega}{N}\right)+\bar{A} \hat{K}_{x}(\omega), G=B+\bar{B} \hat{K}_{u}(\omega) .
\end{aligned}
$$

The roots of the quadratic will depend on $(\theta, \omega)$. If $P^{*}(\theta, \omega)=P^{*}$ is a positive root of (41), then $p$ is determined as

$$
p^{*}=\frac{P^{*} K_{\varepsilon}^{2}}{\rho} .
$$

Note from (41) that since $p^{*}$ does not depend on the volatility parameter $K_{\varepsilon}$, the optimal decision rule (40) does not depend on $K_{\varepsilon}$. Thus the modified certainty equivalence principle related to Hansen and Sargent (2008), which was mentioned above, holds for the distributed parameters linear quadratic regulator problem in the sense that the same decision rules for $\hat{u}(\omega)$ and $\hat{v}(\omega)$ emerge from solving a random version of the appropriate Bellman equation or from a nonstochastic version where $d \hat{\mathcal{B}}(t, \omega) \equiv 0$. However the optimal decision rules depend on the misspecification parameter $C$ as long as $\theta<\infty$. Using this certainty equivalence property, we focus on the nonstochastic 
version of the problem to define hot spots in the space of 'cells' $\mathbb{Z}_{N}$. Hot spots are determined by the interaction of the penalty parameter $\theta$ with $\omega \in \mathbb{Z}_{N}$. We will characterize a hot spot $\omega$ in terms of stability of the state variable in the neighborhood of the FOSS and in terms of low values for welfare reflected in the value function of the problem.

\subsection{Hot spot of type 1: The agglomeration hot spot}

From $(41), \phi(0)=\left[\left(S^{2}-M N\right) / 2 N\right]>0$ by the concavity of the objective. Furthermore the stationary point for (41) will be at $P^{+}=-\gamma_{1} / 2 \gamma_{2}$, while the extremum (maximum or minimum) of $\phi(P)$ will be $\phi\left(P^{+}\right)$We can then distinguish the following cases:

1. $\frac{C^{2}}{\theta}+\frac{G^{2}}{N}<0$ for $\theta<\infty$. In this case $\phi\left(P^{+}\right)$is a maximum and $\phi(P)=0$ has one positive root $P^{*}(\theta, \omega) .^{9}$

2. $\frac{C^{2}}{\theta}+\frac{G^{2}}{N}>0$ for $\theta<\infty$. In this case $\phi\left(P^{+}\right)$is a minimum and $\phi(P)=0$ could have: two positive roots, two negative roots, one positive or one negative root, or no real roots. Furthermore, if:

(a) $\phi\left(P^{+}\right)>0$, there are no real roots.

(b) $\phi\left(P^{+}\right)<0$ and $\phi^{\prime}(0)<0$, there are two positive roots $P_{1,2}^{*}(\theta, \omega)$ or one (double) positive root.

(c) $\phi\left(P^{+}\right)<0$ and $\phi^{\prime}(0)>0$, there are two negative roots $P_{1,2}^{*}(\theta, \omega)$ or one (double) negative root.

We will assume for the rest of this subsection that $\phi(P)=0$ has one positive root $P^{*}(\theta, \omega)$. Then when optimal decision rules are followed, the deterministic state dynamics are:

$$
\begin{aligned}
& \frac{d \hat{x}(t, \omega ; \theta)}{d t}=\left\{\left[A-4 D \sin ^{2}\left(\frac{\pi \omega}{N}\right)+\bar{A} \hat{K}_{x}(\omega)\right]+\right. \\
& \left.\left[B+\bar{B} \hat{K}_{u}(\omega)\right] \frac{2 P^{*}(\omega, \theta) G}{N}+\frac{2 P^{*}(\omega, \theta) C^{2}}{\theta}\right\} \hat{x}(t, \omega) \text { or } \\
& \frac{d \hat{x}(t, \omega ; \theta)}{d t}=\phi(\omega ; \theta) \hat{x}(t, \omega), \hat{x}(0, \omega)=\hat{x}_{0}(\omega) .
\end{aligned}
$$

\footnotetext{
${ }^{9}$ Note that when $\theta \rightarrow \infty$ there are no concerns for misspecification and the regulator trusts the benchmark model.
} 
The solution of (44) in the dual group is $\hat{x}(t, \omega)=\mathcal{A}_{\omega} e^{\phi(\omega) t}$, where $\hat{x}(t, \omega)$ is defined as $\hat{x}(t, \omega)=\hat{x}(\omega, t)-\hat{x}^{*}(\omega)$ by the linearity of the Fourier transform, with $\hat{x}^{*}(\omega)$ being the Fourier transform of the FOSS. Then $A_{\omega}=$ $\hat{x}(0, \omega)-\bar{x}^{*}$ where $\bar{x}^{*}$ is the FOSS and $\hat{x}(0, \omega)$ is the Fourier transform of initial conditions in the neighborhood of the FOSS for all $z .{ }^{10}$

Using the inverse Fourier transform, the solution for the state variable in the primary group is

$$
x(t, z)=\bar{x}^{*}+\sum_{\omega=0}^{N-1} \mathcal{A}_{\omega} \exp \left[\frac{2 \pi i \omega z}{N}+\phi(\omega ; \theta) t\right], z \in \mathbb{Z}_{N},
$$

The evolution of the state variable (45) is very similar to Turing's (1952) formulation regarding morphogenesis associated with chemical substances, although it is derived, in contrast to Turing, from a problem that involves optimization. The part of the exponential $\phi(\omega ; \theta)$ determines the potential instability emerging at frequency or mode $\omega$. If, for some combination of $(\omega, \theta)$, the quantity $\phi(\omega ; \theta)>0$, a wave pattern which becomes more profound with the passage of time emerges. In this case a spatial instability occurs at $(\omega, \theta)$ and agglomeration emerges. In more recent terminology (Murray 2003), $\phi(\omega)$ is a dispersion relationship (see, for example, Brock and Xepapadeas 2008, 2010). A frequency or mode $\hat{\omega}$ will be unstable if $\phi(\hat{\omega} ; \theta)>0$. In this case an optimal agglomeration emerges on the ring.

The interesting result, which is different from previous results on optimaldiffusion-induced or optimal-spillover-induced spatial instability (Brock and Xepapadeas $2008,2009,2010)$, is that instability can be induced by a $\theta<\infty$, while the same instability would not emerge for $\theta \rightarrow \infty$. Thus the following proposition can be stated.

Proposition 1 Assume that when $\theta \rightarrow \infty, \phi(\omega ; \theta)<0$ for all $\omega$ and assume that there exists a critical pair $\left(\omega^{*}, \theta_{1}^{*}\right)$ with $\theta_{1}^{*}<\infty: \phi\left(\omega^{*}, \theta_{1}^{*}\right)>0$. Then optimal robustness induced instability emerges on the ring of cells $\mathbb{Z}_{N}$.

This result suggests the regulator's concerns for model misspecification could induce spatial agglomeration on the ring. This means that the optimal$$
{ }^{10} \hat{x}^{*}(\omega)=\bar{x}^{*}\left(\frac{1}{N} \sum_{z=1}^{N} e^{-2 \pi i \omega \frac{z}{N}}\right)=-\frac{1-e^{-2 i \omega \pi}}{N\left(1-e^{\frac{2 i \omega \pi}{N}}\right)} \bar{x}^{*}=\hat{S}(\omega) \bar{x}^{*}, \omega=0,1, \ldots, N-1 .
$$$$
\text { But } \hat{S}(0)=1 \text { and } \hat{S}(\omega)=0 \text { for } \omega=1, \ldots, N-1 \text {, since } e^{-2 i \omega \pi}=\cos (2 \pi \omega)-i \sin (2 \pi \omega)=1 \text {, }
$$
for $\omega=1, \ldots, N-1$. Thus $\hat{x}^{*}(\omega)=\bar{x}^{*}$.
} 
robust feedback control which will be of the form

$$
u(t, z)=\bar{u}^{*}+\left(\frac{2 P G-S}{N}\right) \sum_{\omega=0}^{N-1} \mathcal{A}_{\omega} \exp \left[\frac{2 \pi i \omega z}{N}+\phi(\omega ; \theta) t\right], z \in \mathbb{Z}_{N}
$$

will also exhibit a wave pattern around the ring. In this case the regulator's concerns about model misspecification induce controls which will break spatial symmetry and produce agglomeration.

\subsection{Hot spot of type 2: The breakdown hot spot}

From (41) let $P^{*}(\theta, \omega)=P^{*}(\theta)(\omega)=P^{*}(\theta, \cdot)$ be the largest root of the quadratic as a function of $\theta$ for each $\omega \in \mathbb{Z}_{N}$. Consider the non-empty sets, assuming they exist, defined as

$$
\Theta(\omega)=\left\{\theta: P^{*}(\theta)(\omega)<0\right\} .
$$

Assume that for some $\omega, P^{*}(\theta)<0$ for a critical $\theta_{c} \in \bar{\Theta}_{\omega} \subset(0,+\infty)$, where $\bar{\Theta}_{\omega}$ is a closed set. Then for this $\omega$ and $\theta \in \bar{\Theta}_{\omega}$, the maximizing agent cannot prevent the minimizing agent from driving the maximizing agent's objective to $-\infty$. Let $\bar{\theta}_{\omega}$ be the maximum $\theta \in \bar{\Theta}_{\omega}$, and consider the set of all the maximum $\theta$ s for each $\omega$ defined as

$$
\Theta_{\omega}^{\max }(\omega)=\left\{\bar{\theta}_{\omega}: P^{*}\left(\bar{\theta}_{\omega}\right)<0, \bar{\theta}_{\omega}=\max \bar{\Theta}_{\omega}\right\} .
$$

We define as a hot spot of type 2 a mode $\omega_{2}$ for which

$$
\omega_{2}: \bar{\theta}_{\omega_{2}}=\max \Theta_{\omega}^{\max }(\omega) .
$$

If we associate the case of $\theta \rightarrow \infty$ with no concern for model misspecification and confidence in the benchmark model, and then interpret reductions in $\theta$ as an increase of concern for model misspecification or lack of confidence in the benchmark model, then a hot spot of type 2 can be given the following interpretation. When $\bar{\theta}_{\omega_{2}}$ is sufficiently far from zero, then at mode $\omega_{2}$, the regulator cannot optimize and cannot prevent her welfare from going to $-\infty$ even though her concerns for misspecification are not very large in the sense of a $\theta$ close to zero. It should be noted that if all sets (47) are empty, then hot spots of type 2 do not exist. 
To provide a concrete example, assume that $S=0$ by a suitable redefinition of variables (Brock and Malliaris 1989, chapter 5), and that $\hat{K}_{x}^{0}(\omega)=$ $\hat{K}_{u}^{0}(\omega)=D=0$, so that we have only nonlocal effects in the state dynamics. A critical value of $\theta$ is defined from (41) as

$$
\theta_{c}:=\frac{-N C^{2}}{G^{2}}, N<0, G=B+\bar{B} \hat{K}_{u}(\omega) .
$$

For $\theta=\theta_{c}$ we have that $P^{*}(\omega)=\frac{M / 2}{2 F-\rho}$ where $F=A+\hat{K}_{x}(\omega)>0$ and $M=q_{11}<0$. Then $P^{*}(\omega)<0$ for a small discount rate. A hot spot of type 2 will be a mode $\omega_{2}$ such that:

$$
\bar{\theta}_{\omega_{2}}=\max \Theta_{\omega}^{\max }(\omega)=\max _{\omega} \frac{-N C^{2}}{\left(B+\bar{B} \hat{K}_{u}(\omega)\right)^{2}} .
$$

It should be noted that the critical $\theta_{c}$ is larger the lower the effectiveness of the control, measured by $G^{2}$, the higher the cost of the control, measured by $N$, and the stronger the impact of misspecification on the state dynamics and the effectiveness of the adversarial agent (Nature), measured by $C^{2}$.

Since by the Plancherel theorem the total value of the regulator's objective is the sum of the values for all modes, the existence of a type 2 hot spot will drive the total value to $-\infty$ and will render regulation useless. If this hot spot does not arise at the spatially homogenous system defined for $D=$ 0 and for $\left(\hat{K}_{x}(\omega), \hat{K}_{h}(\omega)\right)$ fixed numbers independent of $\omega$, then our results suggest that spatial effects and moderate concerns about model misspecification might cause regulation to break down. As we will discuss in the application section, this breakdown might suggest the need to introduce additional regulatory instruments.

\subsection{Hot spot of type 3: The cost of robustness}

However, even if we obtain a positive root $P^{*}(\theta, \omega)$ for all $\omega$, another type of hot spot could emerge. Since the value function can be written as $V(x)=$ $-P^{*} x^{2}$, due to the certainty equivalence, then for a given initial state a large $P^{*}$ corresponds to low welfare and large cost, while a small $P^{*}$ corresponds to higher welfare and smaller cost. Thus if $P^{*} \rightarrow \infty$ then welfare goes to $-\infty$ and cost goes to $+\infty$.

Let $P^{*}(\theta, \omega)>0$. For each $\omega$ let $\theta_{c}^{*}$ be the critical value of $\theta$ for which 
$P^{*}\left(\theta_{c}^{*}, \omega\right)=\max P^{*}(\theta, \omega)$, for all $\theta \in(0,+\infty)$. A hot spot of type 3 will be a mode $\omega_{2}$ such that:

$$
\omega_{3}: P^{*}\left(\theta_{c}^{*}, \omega_{3}\right)=\max _{\omega} P^{*}\left(\theta_{c}^{*}, \omega\right) \text { for all } \omega \in \mathbb{Z}_{N} .
$$

Since $P^{*}\left(\theta_{c}^{*}, \omega_{3}\right)>0$ the regulator can prevent the minimizing agent from driving her objective to $-\infty$, but the regulator will experience low welfare at this point. If $-P^{*}\left(\theta_{c}^{*}, \omega_{3}\right) x^{2}<-P^{*}(\infty, \omega) x^{2}$, then concerns for misspecification reduce the value of the regulator and the largest value reduction occurs at the hot spot $\omega_{3}$. The difference $\left|P^{*}\left(\theta_{c}^{*}, \omega_{3}\right) x^{2}-P^{*}(\infty, \omega) x^{2}\right|$ will provide a measure of the cost of seeking robustness. Since sometimes robust preferences have been associated with a precautionary principle, this robustness cost can be regarded as an indication of the cost of following precautionary policies.

\section{Application: Distributed robust control of a com- mercial fishery}

We illustrate our theory by extending Smith's (1969) well known model of commercial fishing to spatial robustness. We believe that this extension is a new and potentially useful contribution to our paper. We assume that the area of the fishery consists of a ring of $N$ cells so that our space $Z$ is the finite group of integers modulo $N, \mathbb{Z}_{N}$. Let $x(t, z)$ denote biomass at time $t$ and cell $z \in Z$. Fish biomass moves from cell to cell. The movements are short range or local movements which can be described by classic diffusion with diffusion coefficient $D>0$, which means that fish move from cells of high biomass concentration to adjacent cells of low biomass concentration. Let $V(t, z)$ denote the number of identical vessels or firms operating at cell $z$ of the ring, and $h(t, z)$ the harvest rate at cell $z$ per unit time. Thus total harvesting at cell $z$ is $V(t, z) h(t, z)$. The evolution of biomass can then be described as

$$
\begin{aligned}
\frac{\partial x(t, z)}{\partial t} & =f(x(t, z))+E_{d}(t, z)-V(t, z) h(t, z), \\
x(0, z) & =x_{0}(z) \\
E_{d}(t, z) & =D[x(t, z+1)-2 x(t, z)+x(t, z-1)],
\end{aligned}
$$


where $f(x)$ is the recruitment rate or growth function for the fishery, with $f(\underline{x})=f(\bar{x})=0, f^{\prime}\left(x^{0}\right)=0, f^{\prime \prime}(x)<0, x \geq 0,0 \leq \underline{x}<x^{0}<\bar{x}$. When $f(x)$ is quadratic, growth is logistic. Harvested fish is sold at an exogenous world price $p$. The cost per vessel for harvesting rate $h$ is defined as $C(h(t, z), x(t, z), X(t, z), H(t, z)) . X(t, z)=\left(\mathbf{K}_{X} V\right)(t, z), H(t, z)=$ $\left(\mathbf{K}_{h} h\right)(t, z)$ denote nonlocal effects modelled by kernels as defined in section 2. For the cost function we assume, denoting partial derivatives with subscripts, that: (i) $C_{h}>0, C_{h h} \geq 0$; (ii) $C_{x}<0$, which implies resource stock externalities;(iii) $C_{X}>0$, which implies crowding externalities due to congestion effects. We assume that an increase in vessels in a given cell will always increase costs, that is $C_{V}>0$. The kernel formulation in the cost function means that vessels not only in cell $z$ but also near cell $z$ could create congestion effects and increase operating costs of the vessels operating in cell $z$; and (iv) $C_{H}<0$, which implies knowledge or productivity externalities because harvesting that takes place near cell $z$ helps the development of harvesting knowledge in $z$ and reduces operating costs. Profit per vessel at $z$ is defined as $\pi(t, z)=p h(t, z)-C(h(t, z), x(t, z), X(t, z), H(t, z))$. Vessels are attracted to cell $z$ if profits per vessel are positive in this cell. Vessels can be attracted to the ring from locations outside the ring if profits are positive in cells of the ring, so the number of vessels in the ring does not need to be conserved. ${ }^{11}$ Thus the evolution of the vessels is described by:

$$
\begin{aligned}
\frac{\partial V(t, z)}{\partial t} & =\phi[p h(t, z)-C(h(t, z), x(t, z), X(t, z), H(t, z))] \\
\phi & >0, V(0, z)=V_{0}(z)
\end{aligned}
$$

where $\phi$ measures speed of adjustment and is set equal to one without loss of generality. A regulator is trying to determine an optimal level of harvesting per vessel in each cell. This harvesting level can be used, for example, to set up a quota system in each cell of the ring. The regulator's objective is the maximization of discounted profits over the whole ring by taking into account biomass diffusion as well as stock, congestion and knowledge

\footnotetext{
${ }^{11}$ To simplify we ignore transportation costs.
} 
externalities. ${ }^{12}$. The regulator's objective is therefore

$$
\max _{\{h(t, z)\}} \sum_{z \in Z}\left\{\int_{0}^{\infty} e^{-\rho t} V(t, z)[p h(t, z)-C(h(t, z), x(t, z), X(t, z), H(t, z))] d t\right\} .
$$

The regulator however has concerns regarding the specification of biomass dynamics in each cell. These concerns are captured by a deterministic specification error which is expressed in terms of deviations from the benchmark case which is defined for $v(t, z):=0$. The specification error is distributed across the domain $Z$ according to $v(t)(z)$, so that the error may vary across cells at the same point in time. This assumption means that, depending on her scientific knowledge, the regulator might trust the benchmark model more or less depending on the cell. For a large enough ring, this assumption - which implies spatially differentiated degrees of scientific uncertainty - seems plausible. When the model is misspecified, the benchmark model remains a good approximation so the misspecification error satisfies (2). Each cell of the fishery is also subject to a stochastic force represented by a Wiener process which is placed in each cell as described in section 2. Under deterministic misspecification and stochastic shocks, the biomass evolution is described by

$$
\frac{\partial x(t, z)}{\partial t}=f(x(t, z))+E_{d}(t, z)-V(t, z) h(t, z)+C v(t, z)+(\mathbf{K} d \mathcal{B})(t, z) .
$$

The regulator's concerns about model misspecification are incorporated into robust preferences. Thus the regulator decides about optimal harvesting per vessel in each cell, by solving a problem where Nature will play the role of the minimizing or 'mean' agent. In this context the regulator considers that Nature 'chooses' a misspecification error to minimize the regulator's objective and, by doing so, Nature determines lower bounds to the performance of the regulation. If the lower bound tends to $-\infty$, then regulation is useless. The problem of the regulator is therefore the distributed parameter robust control problem with local and nonlocal spatial effects of the type

\footnotetext{
${ }^{12}$ To simplify the interpretation of results and the analysis, we do not include existence values for the biomass.
} 
described in sections 2 and 3 , which can be written, dropping $(t, z)$ in some places to simplify notation, as:

$$
\begin{gathered}
\sup _{h(t, z)} \inf _{v(t, z)} E_{0} \sum_{z \in \mathbb{Z}_{N}}\left\{\int_{0}^{\infty} e^{-\rho t}[V[p h-C(h, x, V, H)]\right. \\
\left.\left.+\theta v^{2}(t, z)\right] d t\right\}
\end{gathered}
$$

subject to (55), (57).

Let $\left(\bar{x}^{*}, \bar{V}^{*}, \bar{h}^{*}, 0, \bar{\lambda}^{*}, \bar{\mu}^{*}\right)$ be a FOSS for the spatially independent deterministic benchmark model as defined in section 3 and appendix 1 , with $(\lambda, \mu)$ the costate variables associated with the spatially independent deterministic benchmark dynamics corresponding to (55), (57) respectively. Assume that this FOSS has the local saddle point property. Linear quadratic approximation, application of the discrete Fourier transform and the Plancherel theorem, and use of the certainty equivalence property as described in section 3, allow us to write the linear quadratic approximation of problem (58) around the FOSS as a set of countable finite dimensional linear quadratic problems, one problem for each $\omega$ in the dual space $\mathbb{Z}_{N}$. The regulator's objective now is to determine an optimal harvesting rule that takes into account misspecifications concerns in the neighborhood of this FOSS.

$$
\begin{aligned}
& \sup _{\hat{h}(t, z)} \inf _{\hat{v}(t, z)} \sum_{\omega \in \mathbb{Z}_{N}}\left[\int_{0}^{\infty} e^{-\rho t} \frac{1}{2}\left[\hat{w}^{\prime} \hat{Q} \hat{w}+\theta \hat{v}^{2}(t, \omega)\right] d t\right] \\
\hat{w}^{\prime}= & (\hat{x}(t, \omega), \hat{V}(t, \omega), \hat{h}(t, \omega))^{\prime}, \hat{Q}=\left[\hat{q}_{i j}(\omega)\right] \quad i, j=1,2,3 \\
& \frac{d \hat{x}(t, \omega)}{d t}=A_{1} \hat{x}(t, \omega)+A_{2} \hat{V}(t, \omega)+A_{3} \hat{h}(t, \omega)+ \\
& C \hat{v}(t, \omega), \hat{x}(0, \omega)=\hat{x}_{0}(\omega) \\
& \frac{d \hat{V}(t, \omega)}{d t}=B_{1} \hat{x}(t, \omega)+B_{2} \hat{h}(t, \omega), \hat{V}(0, \omega)=\hat{V}_{0}(\omega) \\
A_{1}= & {\left[f_{x}^{*}-4 D \sin ^{2}\left(\frac{\pi \omega}{N}\right)\right], A_{2}=\bar{h}^{*}, A_{3}=\bar{V}^{*} } \\
B_{1}= & -\phi\left(C_{x}^{*}+C_{X}^{*} \hat{\mathbf{K}}_{x}(\omega)\right), B_{2}=\phi\left(p-C_{h}^{*}-C_{H}^{*} \hat{\mathbf{K}}_{h}(\omega)\right)
\end{aligned}
$$

where $\left({ }^{*}\right)$ when associated with partial derivatives indicates derivative evaluated at the FOSS, matrix $\hat{Q}$ is negative definite and its elements $\hat{q}_{i j}(\omega)$ can be calculated using the procedure described in appendix 2. Note that the coefficients of the transition equations depend on local and nonlocal spatial effects in the frequency domain. Assuming a quadratic value function 
$W(\hat{x}(\omega, \theta), \hat{V}(\omega, \theta))=-P_{1} x^{2}-P_{2} V^{2}-P_{3} x V$ and following the procedure of section 3 , we obtain the optimal feedback controls as:

$$
\begin{aligned}
\hat{v}^{*}(\omega, \theta) & =\frac{C\left(2 P_{1} \hat{x}(\omega, \theta)+P_{3} \hat{V}(\omega, \theta)\right)}{\theta} \\
\hat{h}^{*}(\omega, \theta) & =\frac{1}{\hat{q}_{33}}\left[\left(2 P_{1} A_{3}+P_{3} B_{2}-\hat{q}_{31}\right) \hat{x}(\omega, \theta)+\left(2 P_{2} B_{2}+P_{3} A_{3}-\hat{q}_{32}\right) \hat{V}(\omega, \theta)\right] .
\end{aligned}
$$

Substituting the optimal feedback controls into the value functions and equating coefficients of the same power, the parameters of the value function are obtained as the solution of a nonlinear system in $\left(P_{1}, P_{2}, P_{3}\right)$ which has the structure ${ }^{13}$

$$
\begin{aligned}
\eta_{1}\left(P_{2}, P_{3}, P_{2}^{2}, P_{3}^{2}\right)+\frac{C^{2}}{2 \theta} P_{3}^{2} & =0 \\
\eta_{2}\left(P_{1}, P_{2}, P_{3}, P_{3}^{2}\right)+\frac{2 C^{2}}{\theta} P_{1} P_{3} & =0 \\
\eta_{3}\left(P_{1}, P_{3}, P_{1}^{2}, P_{3}^{2}\right)+\frac{2 C^{2}}{\theta} P_{1}^{2} & =0 .
\end{aligned}
$$

We note the following: When the regulator is not concerned about model misspecification, then $\theta \rightarrow \infty$ and our problem is a distributed parameter control problem. Local spatial effects are captured by the term $4 D \sin ^{2}\left(\frac{\pi \omega}{N}\right)$ which reflects biomass diffusion, while nonlocal effects are captured by the terms $\left(\hat{\mathbf{K}}_{x}(\omega), \hat{\mathbf{K}}_{h}(\omega)\right)$ which reflect congestion and knowledge effects. When spatial effects are not present and $\theta \rightarrow \infty$, then our problem is a standard linear quadratic regulator problem. A solution of (67)-(69) will provide the parameters of the value function in the frequency domain as functions of $\theta$ and the local and the nonlocal spatial effects, or

$$
P_{i}^{*}(\omega)=P_{i}^{*}\left(\omega, \theta, D, \hat{\mathbf{K}}_{x}(\omega), \hat{\mathbf{K}}_{h}(\omega)\right)
$$

This solution can be used to locate sufficient conditions for hot spots of type 1-3 discussed above.

\subsection{Agglomeration hot spot (type 1)}

To study this hot spot we assume that $P_{1}^{*}<0, P_{2}^{*}<0, P_{1}^{*} P_{2}^{*}-\left(P_{3}^{*}\right)^{2}>$ 0 so that the value function is concave. Then the state dynamics when

\footnotetext{
${ }^{13}$ The full system is presented in appendix 3 .
} 
the maximizing agent (regulator) and the minimizing agent (Nature) make optimal choices can be written as

$$
\begin{aligned}
\left(\begin{array}{c}
\frac{d \hat{x}(t, \omega)}{d t} \\
\frac{d \hat{V}(t, \omega)}{d t}
\end{array}\right)= & \mathcal{A}\left(\begin{array}{c}
\hat{x}(t, \omega) \\
\hat{V}(t, \omega)
\end{array}\right), \mathcal{A}=\left[\alpha_{i j}\right], i, j=1,2 \\
\alpha_{11}= & f_{x}^{*}-4 D \sin ^{2}\left(\frac{\pi \omega}{N}\right)+\frac{\bar{V}^{*}}{\hat{q}_{33}}\left(2 P_{1}^{*} A_{3}+P_{3}^{*} B_{2}-\hat{q}_{31}\right)(72) \\
\alpha_{12}= & \bar{h}^{*}+\frac{\bar{V}^{*}}{\hat{q}_{33}}\left(2 P_{2}^{*} B_{2}+P_{3}^{*} A_{3}-\hat{q}_{32}\right)+\frac{C^{2} P_{3}^{*}}{\theta} \\
\alpha_{21}= & -\phi\left(C_{x}^{*}+C_{X}^{*} \hat{K}_{x}(\omega)\right)+ \\
& \frac{B_{2}}{\hat{q}_{33}}\left(2 P_{1}^{*} A_{3}+P_{3}^{*} B_{2}-\hat{q}_{31}\right) \\
\alpha_{22}= & \frac{\phi}{\hat{q}_{33}}\left(p-C_{h}^{*}-C_{H}^{*} \hat{K}_{h}(\omega)\right)\left(2 P_{2}^{*} B_{2}+P_{3}^{*} A_{3}-\hat{q}_{32}(75)\right.
\end{aligned}
$$

For stability of the FOSS in all frequencies $\omega \in \mathbb{Z}_{N}$ we need the two eigenvalues of matrix $\mathcal{A}$ denoted by $\left(\lambda_{1}, \lambda_{2}\right)$ to be real and negative or to have negative real parts for all $\theta$. Let $\lambda_{1}$ denote the largest eigenvalue of matrix $\mathcal{A}$. Then the following proposition can be stated:

Proposition 2 (i) If $\lambda_{1}>0$ for a set of frequencies $\Omega \in \mathbb{Z}_{N}$ when $\theta \rightarrow \infty$ then an agglomeration hot spot exists for frequencies or modes $\omega \in \Omega$, where $\Omega$ can be a singleton. The agglomeration hot spot is independent of concerns for model misspecification. (ii) If $\lambda_{1}>0$ for a set of frequencies $\Omega \in \mathbb{Z}_{N}$ if and only if $\theta \in[\underline{\theta}, \bar{\theta}]$, with $\bar{\theta}<\infty$, then an agglomeration hot spot is induced by the regulator's concerns about model misspecification.

An agglomeration hot spot in this context means that optimal regulation implies the generation of a heterogenous spatial pattern of fish biomass and fishing vessels along the ring, with the form of a wave pattern. These patterns will be realized in the primal space $\mathbb{Z}_{N}$ when inverse Fourier transforms similar to (45) are applied. Furthermore, optimal harvesting, since it is a feedback function of fish biomass and vessels, is also going to exhibit a similar wave heterogenous spatial pattern. Thus if quotas are to be issued, the amount of quotas will be different for each cell of the ring and the approximate optimal spatiotemporal quota path will be $\left\{h^{*}(t, z)\right\}_{z=1}^{Z=N}$. Suppose that $h^{*}\left(t, z_{1}\right)<h^{*}\left(t, z_{2}\right)$, then if quotas can be traded across cells, the optimal trading ratio will be $h^{*}\left(t, z_{1}\right) / h^{*}\left(t, z_{2}\right)$ for quotas of cell 2 to be 
used for harvesting in cell 1. The importance of part (ii) of proposition 2 is that the spatially heterogeneous quota pattern can be induced by concerns about model misspecification, since reduction of $\theta$ means increase in the regulator's concerns about model misspecification. To put it in more general terms, when concerns about possible misspecifications of state dynamics differ across sites, then the regulator might introduce spatially differentiated instruments and generate agglomerations.

When it is optimal to generate agglomeration through the mechanism described above, the question of what will be the final - or the steady state equilibrium - agglomeration, which is the spatial pattern of vessels and fish biomass after a long lapse of time, arises. Emergence of agglomeration implies that the spatial instability will tend to become 'catastrophic' in the sense that the amplitude of the waves increase with time. This pattern will be halted, however, when the fish biomass in some cells becomes zero. This is because in the dynamic system of fish biomass and vessels (57), (55), biomass acts as an activator, since an increase in biomass in a cell will reduce costs and increase the rate of growth of vessels in this cell, while vessels act as an inhibitor, since an increase in the number of vessels in a cell will reduce the rate of growth of biomass in this cell. Thus when biomass collapses in a cell, cost per vessel will become very high, profits per vessel will become negative and number of vessels in this cell will eventually decline to zero. Whether biomass diffusion will increase the stock of fish in the cell to the extent that vessels will be attracted depends on the specific structure of the fishery, but this is a possibility suggesting that quite complex spatiotemporal patterns might emerge in the long run. Although the analysis of the equilibrium spatial distribution of biomass, vessels and quotas is beyond the purpose of the present paper, it can be approximated by substituting the optimal harvesting rule $\left\{h^{*}(t, z)\right\}_{z=1}^{Z=N}$ in feedback form into the system of (57), (55) and then solving the system with $(\partial x / \partial t)=(\partial V / \partial t)=0$. This will be a system of difference equations in the spatial dimension with circle boundary conditions. In principle numerical schemes can be used to provide a description of equilibrium distributions. 


\subsection{Break down hot spot (type 2)}

Consider the non-empty sets, assuming they exist, defined as

$$
\begin{aligned}
\Theta(\omega)= & \left\{\theta: \text { all } P_{i}^{*}(\theta) \in \mathbb{R}, i=1,2,3 \text { which are solutions of }(67)-(69)\right. \\
& \text { imply a convex value function } W(\hat{x}(\omega, \theta), \hat{V}(\omega, \theta))\} .
\end{aligned}
$$

These sets represent $\theta^{\prime}$ s at which the value function is convex. A mode $\omega_{2}$ will be a hot spot of type 2 if the value function becomes convex at this mode for the largest $\theta<\infty$. If such a hot spot exists, the regulator cannot prevent her value from going to $-\infty$ at this mode which means that she cannot prevent the performance of the regulation by a quota system from reaching $-\infty$. Since by the Plancherel theorem the total value of the regulator's objective is the sum of the values for all modes, the existence of a break down hot spot will drive the total value to $-\infty$ and will render regulation useless. If this hot spot does not arise at the spatially homogenous system defined for $D=$ 0 and $\left(\hat{K}_{x}(\omega), \hat{K}_{h}(\omega)\right)$ independent of $\omega$, then our results suggest that spatial effects and moderate concerns about model misspecification might cause regulation to break down. Although the complexity of the model does not allow analytical results, numerical simulation might be possible to reveal the relative contribution of local and nonlocal spatial effects to this break down. Identification of this contribution might be important for refining regulation. If, for example, nonlocal congestion effects are responsible for the emergence of this hot spot, then new regulatory instruments, such as entry licences to a cell, could be introduced to prevent these effects from creating the hot spot.

\subsection{The cost of robustness hot spot (type 3 )}

A type 3 hot spot is consistent with a concave value function but corresponds to a mode $\omega$ and a parameter $\theta$ at which the value function has the smallest value for any given initial state of fish biomass and vessels. Let, for all $\theta \in(0,+\infty)$,

$$
\left(P_{1}^{*}\left(\theta_{c}^{*}, \omega\right), P_{2}^{*}\left(\theta_{c}^{*}, \omega\right), P_{3}^{*}\left(\theta_{c}^{*}, \omega\right)\right)=\max \left\|P_{1}^{*}\left(\theta_{c}, \omega\right), P_{2}^{*}\left(\theta_{c}, \omega\right), P_{3}^{*}\left(\theta_{c}, \omega\right)\right\|,
$$


then the mode $\omega_{3}$ that maximizes $\left\|P_{1}^{*}\left(\theta_{c}^{*}, \omega\right), P_{2}^{*}\left(\theta_{c}^{*}, \omega\right), P_{3}^{*}\left(\theta_{c}^{*}, \omega\right)\right\|$ will be a type 3 hot spot. At this hot spot regulation does not break down but the largest reduction of value occurs. Since concerns about model misspecification have been associated with the concept of a precautionary principle, our result can be used to characterize costs or benefits from precaution. Since the

no concern case corresponds to the value function $W(\hat{x}(\omega, \infty), \hat{V}(\omega, \infty))$, the cost or benefits from precaution can be determined by

$$
W\left(\hat{x}\left(\omega_{3}, \infty\right), \hat{V}\left(\omega_{3}, \infty\right)\right)-W\left(\hat{x}\left(\omega_{3}, \theta_{c}\right), \hat{V}\left(\omega_{3}, \theta_{c}\right)\right)
$$

As in the case of the break down hot spot discussed above, identification of the relative contribution of local and nonlocal effects might be important for refining regulation and preventing large losses in value due to the application of a precautionary principle.

\section{Conclusions and suggestions for future research}

This paper has developed robust control theory in spatial settings by building on recent work on distributed control of spatially invariant systems (Bamieh et al. 2002; Curtain et al. 2008; Brock and Xepapadeas 2008, 2009, 2010) and on robust control in economics (Salmon 2002; JET 2006; Hansen and Sargent 2008). By adapting and extending this work, we produced a linear quadratic approximation to this problem (see Appendix 1). Using that linear quadratic approximation, we were able to decompose an apparently intractable infinite horizon robust control linear quadratic problem on an infinite dimensional space with highly coupled spatial dynamics into a countable number of tractable finite dimensional infinite horizon robust control linear quadratic problems. Using these finite dimensional problems, we were able to characterize the robust solution for the original infinite dimensional linear quadratic problem. As far as we know, this approach to spatial robust control is new to economics. Our approach provides closed form solutions to a wide class of spatial robust control problems in economics. Our approach also leads to a useful precise formulation of three types of "hot spots".

Hot spots of type 1 are spatial agglomerations induced by concerns of the optimizing agent about model misspecification. Here the penalty parameter 
$\theta$ for choices of $v(t, z)$ by the adversarial agent is still larger than any breakdown point $\underline{\theta}(\omega), \omega \in \hat{\mathbb{Z}}_{N}$ and is still larger than any point where the value function of the maximizing agent loses concavity in the maximizing agent's state variable. While the linear quadratic approach can signal the existence of such hot spots, the accuracy of the linear quadratic approximation will break down and underlying nonlinearities left out of the linear quadratic approximation of the problem determine the ultimate patterns. This task is beyond the scope of the current paper and is reserved for future research.

Hot spots of type 2 occur when the penalty parameter, $\theta$, for the adversarial agent is lowered from $+\infty$ to the "first" value of $\theta$ where there is an $\omega$ where the adversarial agent can drive the maximizing agent's welfare to $-\infty$. This kind of hot spot suggests a new type of precautionary principle that operates when model uncertainty is present. Recall that the size of $\theta$ is inversely related to the size of the model uncertainty set (e.g., Hansen and Sargent 2008, chapter 2). Thus the optimizing agent will want to invest a lot of resources in reducing model uncertainty that the regulator wishes to robustify against when type 2 hot spots exist. Hot spots of type 2 guide these resources towards reduction of model uncertainty at the particular where the hot spot exists. We plan to explore this type of precautionary principle as well as to develop the ways in which to formulate the problem of optimal allocation of model uncertainty reduction resources in spatial settings where the concept of "space" is much broader than physical space.

Hot spots of type 3 occur at $\omega$ 's where the value function in the Fourier domain computed from (37) and the text following (37), call it $\hat{W}\left(\hat{x}_{0}(\omega), \omega ; \theta\right)$, is particularly low, i.e., when its absolute value is particularly large at a particular level of model uncertainty reflected by a particular value of $\theta$. Again, this type of hot spot reveals not only a strong incentive to employ resources to learn more about the system in order to reduce model uncertainty, but also directs allocation of those resources, much as indicated by hot spots of type 2 .

Last but not least, we apply our approach to a spatial extension of a classical work in environmental economics and bioeconomics, Vernon Smith's (1969) model of commercial fishing. We take the linear quadratic approximation around a flat optimal steady state where each site has an equal number of vessels, using the material in the Appendix. We then study the analytics of the solution and the three basic types of hot spots. We locate 
sufficient conditions for when it is optimal to induce agglomeration at some sites independently of concerns about model misspecification. We also locate sufficient conditions under which concerns about model misspecification and robustification against it lead to creation of "precautionary" agglomerations. This is a novel (to our knowledge) form of precautionary principle. Of course a linear quadratic approach can only signal that the FOSS is optimally (or robustly optimally) unstable. A study of the full nonlinear problem is needed to assess whether agglomerations are actually created or whether some other type of pattern is created. It is beyond the scope of the current paper to conduct this study. This study would be the optimal control analog of studies in mathematical biology and elsewhere of the actual nonlinear patterns created when the linearization approach signals instability. In Brock and Xepapadeas (2008) and (2010), we used numerical methods to compute the optimal aggregations when the linear quadratic approach signaled instability of the FOSS. But we did not do robust control. It is beyond the scope of the current paper to do an analog of the Brock and Xepapadeas computational analysis for the robust control problems studied here. But we conjecture that it will be a relatively straightforward adaptation of the methods of Brock and Xepapadeas.

We placed the dynamics in this paper upon a finite ring of cells, i.e., the "primary" group $\mathbb{Z}_{N}$ with modulo $N$ arithmetic where Fourier transforms lie in the "dual" group $\hat{\mathbb{Z}}_{N}=\mathbb{Z}_{N}$. We did this to present the analytical results in bold relief. We conjecture that the methods developed here can be extended to many other pairs of primary and dual groups. We further conjecture that the notation will become more complex but the basic methods will be the same. See, for example, Bamieh et al. (2002, page 1092 and following material, e.g. Table I) for the wide variety of settings that may be treated in the context of spatially distributed control. This makes it clear that in the context of spatially distributed control it will be basically a matter of more complex notation, especially for two dimensional or higher dimensional spaces. Hence, this is why we conjecture that the same will hold for robust control. We leave this extension to future research.

Appendix

Appendix 1: Linear quadratic (LQ) approximation of nonlinear distributed parameter penalty robust control problem

We extend the general approach set out by Magill (1977a,b) and we 
consider a general nonlinear distributed parameter penalty robust control problem with deterministic misspecification only, since the modified certainty equivalence property will apply to the LQ problem. We will deal with a general distributed parameter problem where space is continuous. State and control functions can be identified with the abstract functions $x(t)(z)=x(t, \cdot), u(t)(z)=u(t, \cdot)$ which take values on $Z$ and which belong to the space of vector valued functions which are square integrable with respect to the Haar measure, while the deterministic misspecification is again the real function $v(t, z)$ which is identified with the abstract function $v(t)(z)=v(t, \cdot)$ that takes values into the space of functions which are square integrable with respect to the Haar measure. In deriving the LQ approximation we use a continuous finite space formulation with circle boundary condition to simplify the exposition. Our results can be extended to a discrete space $\mathbb{Z}_{N}$. Let the nonlinear penalty robust control problem:

$$
\begin{aligned}
& \max _{u(t, z)} \min _{v(t, z)} \int_{z \in Z} \int_{0}^{\infty} e^{-\rho t}\left[f_{0}\left(x(t, z), u(t, z),\left(\mathbf{K}_{0} x\right)(t, z),\left(\mathbf{K}_{0} u\right)(t, z)\right)\right. \\
& \left.+\theta v^{2}(t, z)\right] d t d z
\end{aligned}
$$

subject to

$$
\begin{array}{r}
\frac{\partial x(t, z)}{\partial t}=f(x(t, z), u(t, z),(\mathbf{K} x)(t, z),(\mathbf{K} u)(t, z), v(t, z))+\quad(80) \\
D \frac{\partial^{2} x}{\partial z^{2}}, x(0, z)=x_{0}(z) \text { and circle boundary conditions }
\end{array}
$$

$$
\begin{gathered}
\left(\mathbf{K}_{0} \phi\right)(t, z):=\int_{Z} K_{j}^{0}\left(z-z^{\prime}\right) \phi\left(t, z^{\prime}\right) d z^{\prime}=\Phi(t, z), j, \phi=x, u, \Phi=X_{0}, U_{0} \\
(\mathbf{K} \phi)(t, z):=\int_{Z} K_{j}\left(z-z^{\prime}\right) \phi\left(t, z^{\prime}\right) d z^{\prime}=\Phi(t, z), j, \phi=x, u, \Phi=X, U
\end{gathered}
$$

Let

$\mathcal{H}\left(x, u, v, X_{0}, U_{0}, X, U, \lambda, D\right)=f_{0}\left(x, u, X_{0}, U_{0}\right)+\theta v^{2}+\lambda\left[f(x, u, X, U, v)+D \frac{\partial^{2} x}{\partial z^{2}}\right]$

be the current value Hamiltonian for the distributed parameter penalty robust control problem (79), and assume that an optimal solution to this problem exists with the maximizing and the minimizing agents taking simultaneous decisions. Let $u^{*}(t, z), v^{*}(t, z)$ denote the optimal controls and 
$x^{*}(t, z), \lambda^{*}(t, z)$ denote the corresponding optimal state and costate paths respectively.

A special case of this optimal solution is the optimal solution to the spatially independent deterministic benchmark problem. This problem is a special case of problem (79) for $v(t, z) \equiv 0, D=0, x(t, z)=x(t), u(t, z)=$ $u(t)$. Spatial independence means that the kernel operators can be written as $(\mathbf{\Phi} \phi)(t, z)=\phi(t) \int_{z^{\prime} \in Z} \varphi\left(z-z^{\prime}\right) d z^{\prime}$, where $\int_{z^{\prime} \in Z} \varphi\left(z-z^{\prime}\right) d z^{\prime}=\bar{\varphi}$ is a fixed parameter for given $\varphi(\cdot)$, and $\phi=(x, u), \varphi=\left(\mathbf{K}_{x}^{0}, \mathbf{K}_{x}, \mathbf{K}_{u}^{0}, \mathbf{K}_{u}\right)$. Thus at the spatially independent model, kernels are fixed parameters. The current value Hamiltonian for this problem is simply:

$$
H(x, \lambda, u)=f_{0}(x, u)+\lambda f(x, u)
$$

where $\lambda(t)$ is the costate variable. From the maximum principle, the solution of the problem is determined by the system

$$
\begin{aligned}
H_{u}(x, \lambda, u) & =0 \\
H_{x}(x, \lambda, u)+\dot{\lambda}-\rho \lambda & =0 \\
H_{\lambda}(x, \lambda, u) & =0 .
\end{aligned}
$$

Let $\left(\bar{x}^{*}, \bar{u}^{*}, 0, \bar{\lambda}^{*}\right)$ be a steady state of the spatially independent benchmark model corresponding to paths that satisfy transversality conditions at infinity. We shall call this steady state a flat optimal steady state and we will assume that the process described by (80) starts close to the FOSS, or that $x(0, z)$ starts close to $x^{*}$ for all $z \in Z$.

Let

$$
\begin{aligned}
& (\chi, \gamma, \zeta, \eta)= \\
& \left(x(t, z)-x^{*}(t, z), u(t, z)-u^{*}(t, z), v(t, z)-v^{*}(t, z), \lambda(t, z)-\lambda^{*}(t, z)\right)
\end{aligned}
$$

denote deviations of the paths for the state, control and costate functions from the optimal paths. Deviation should be understood as functions $\chi(t)(z)=$ $\chi(t, \cdot), \gamma(t)(z)=\gamma(t, \cdot), \zeta(t)(z)=\zeta(t, \cdot), \eta(t)(z)=\eta(t, \cdot)$ which take values on $Z$ and which belong to the space of vector valued functions which are square integrable with respect to the Haar measure. A special case of these deviations are deviations from the FOSS $\left(\bar{x}^{*}, \bar{u}^{*}, 0, \bar{\lambda}^{*}\right)$.

Perturb the optimal controls by letting 


$$
\begin{aligned}
& u(t, z)=u^{*}(t, z)+\varepsilon \gamma(t, z) \\
& v(t, z)=v^{*}(t, z)+\varepsilon \zeta(t, z) .
\end{aligned}
$$

For a control of the form (87), (88) we adapt Athans and Falb (1966, page 261) to focus on perturbations of the state function of the form below,

$$
x(t, z)=x^{*}(t, z)+\varepsilon y(t, z)+\varepsilon^{2} \xi(t, z)+o\left(\varepsilon^{2}, t, z\right),
$$

where $y$ and $\xi$ are first- and second-order state perturbations respectively, $o\left(\varepsilon^{2}, t, z\right)$ is defined in the $L^{2}$ norm sense $o\left(\varepsilon^{2}, t, z\right) \rightarrow 0$ as $\varepsilon^{2} \rightarrow 0$ uniformly in $(t, z)$. Athans and Falb (1966, pp. 254-265) show that control perturbations of the form (87) lead to state perturbations of the form (89) under appropriate regularity conditions for the case where $Z$ is one point. Substituting the perturbed state and controls into the kernel expressions and using the linearity of the integral operator we obtain

$$
\begin{aligned}
(\mathbf{K} x)(t, z)= & \mathbf{K}\left(x^{*}(t, z)+\varepsilon y(t, z)+\varepsilon^{2} \xi(t, z)+o\left(\varepsilon^{2}, t, z\right)\right)=(90) \\
& \left(\mathbf{K} x^{*}\right)(t, z)+\varepsilon(\mathbf{K} y)(t, z)+\varepsilon^{2}(\mathbf{K} \xi)(t, z)+\mathbf{K} o\left(\varepsilon^{2}, t, z\right) \\
(\mathbf{K} u)(t, z)= & \mathbf{K}\left(u^{*}(t, z)+\varepsilon \gamma(t, z)\right)=\left(\mathbf{K} u^{*}\right)(t, z)+\varepsilon(\mathbf{K} \gamma)(t, z) .(91)
\end{aligned}
$$

We substitute perturbed control, state and kernels into (80) and then expand it as a Taylor series around the FOSS $\left(\bar{x}^{*}, \bar{u}^{*}, 0, \bar{\lambda}^{*}\right)$, where the expansion is defined in terms of Fréchet derivatives of $f(\cdot)$. Omitting $(t, z)$ to ease notation and letting $\left({ }^{*}\right)$ denote evaluation at the FOSS we obtain

$$
\begin{aligned}
& \varepsilon \frac{\partial y}{\partial t}+\varepsilon^{2} \frac{\partial \xi}{\partial t}=f\left(\bar{x}^{*}, \bar{u}^{*}, \bar{x}^{*} \overline{\mathbf{K}}_{x}, \bar{u}^{*} \overline{\mathbf{K}}_{u}, 0\right)+f_{x}^{*}\left[\varepsilon y+\varepsilon^{2} \xi+o\left(\varepsilon^{2}\right)\right]+f_{u}^{*} \varepsilon \gamma+ \\
& f_{X}^{*}\left[\varepsilon(\mathbf{K} y)(t, z)+\varepsilon^{2}(\mathbf{K} \xi)(t, z)+\mathbf{K} o\left(\varepsilon^{2}, t, z\right)\right]+f_{U}^{*} \varepsilon(\mathbf{K} \gamma)(t, z) \\
& f_{v}^{*} \varepsilon \zeta+\varepsilon D \frac{\partial^{2} y}{\partial z^{2}}+\varepsilon^{2} D \frac{\partial^{2} \xi}{\partial z^{2}}+\frac{1}{2} w^{\prime} W w+\text { higher order terms, }
\end{aligned}
$$

where $\overline{\mathbf{K}}_{x}, \overline{\mathbf{K}}_{u}$ are the fixed, at the FOSS, kernels and $w^{\prime} W w$ is the quadratic form of the second order Fréchet derivatives of $f(\cdot)$ with

$$
w=\left(\varepsilon y+\varepsilon^{2} \xi, \varepsilon \gamma, \varepsilon(\mathbf{K} y)+\varepsilon^{2}(\mathbf{K} \xi), \varepsilon(\mathbf{K} \gamma), \varepsilon \zeta\right) .
$$

Noting that $f\left(\bar{x}^{*}, \bar{u}^{*}, \bar{x}^{*} \overline{\mathbf{K}}_{x}, \bar{u}^{*} \overline{\mathbf{K}}_{u}, 0\right)=0$ since it is evaluated at the FOSS, 
dividing throughout by $\varepsilon$ and then taking the limit as $\varepsilon \rightarrow 0$, we obtain:

$$
\frac{\partial y}{\partial t}=f_{x}^{*} y+f_{u}^{*} \gamma+f_{X}^{*}(\mathbf{K} y)+f_{U}^{*}(\mathbf{K} \gamma)+f_{v}^{*} \zeta+D \frac{\partial^{2} y}{\partial z^{2}} .
$$

Using the Hamiltonian function (81) write

$$
\begin{aligned}
\mathcal{H}\left(x, u, v, X_{0}, U_{0}, X, U, \lambda^{*}\right) & =\mathcal{G}\left(x, u, v, X_{0}, U_{0}, X, U, \lambda^{*}\right)+\lambda^{*} D \frac{\partial x^{2}}{\partial z^{2}} \\
\mathcal{G}\left(x, u, v, X_{0}, U_{0}, X, U, \lambda^{*}\right) & =f_{0}\left(x, u, X_{0}, U_{0}\right)+\theta v^{2}+\lambda^{*} f(x, u, X, U, v) .
\end{aligned}
$$

Then the performance functional for (79) along the optimal path can be written as

$J\left(u^{*}, v^{*}\right)=\int_{z \in Z} \int_{0}^{\infty} e^{-\rho t}\left[\mathcal{G}\left(x^{*}, u^{*}, v^{*}, X_{0}^{*}, U_{0}^{*}, X^{*}, U^{*}, \lambda^{*}\right)+\lambda^{*} D \frac{\partial^{2} x^{*}}{\partial z^{2}}-\lambda^{*} \frac{\partial x^{*}}{\partial t}\right] d t d z$

with $(t, z)$ omitted to ease notation. The performance functional along a perturbed path, with perturbations given by (87), (88), (89), which is evaluated along the $\lambda^{*}(t, z)$ path, is

$$
J(u, v)=\int_{z \in Z} \int_{0}^{\infty} e^{-\rho t}\left[\mathcal{G}\left(x, u, v, X_{0}, U_{0}, X, U, \lambda^{*}\right)+\lambda^{*} D \frac{\partial x^{2}}{\partial z^{2}}-\lambda^{*} \frac{\partial x}{\partial t}\right] d t d z .
$$

Let $\mathcal{G}^{0}=\mathcal{G}\left(x^{*}, u^{*}, v^{*}, X_{0}^{*}, U_{0}^{*}, X^{*}, U^{*}, \lambda^{*}\right)$, then

$$
J(u, v)-J\left(u^{*}, v^{*}\right)=\int_{z \in Z} \int_{0}^{\infty} e^{-\rho t}\left[\left(\mathcal{G}-\mathcal{G}^{0}\right)-\lambda^{*} \frac{\partial\left(x-x^{*}\right)}{\partial t}-\lambda^{*} D \frac{\partial^{2}\left(x-x^{*}\right)}{\partial z^{2}}\right] d t d z .
$$

The terms $\lambda^{*} \frac{\partial\left(x-x^{*}\right)}{\partial t}$ and $\lambda^{*} D \frac{\partial^{2}\left(x-x^{*}\right)}{\partial z^{2}}$ can be transformed as follows:

Integrating by parts the term $\int_{0}^{\infty} e^{-\rho t} \lambda^{*} \frac{\partial\left(x-x^{*}\right)}{\partial t} d t$ and using appropriate temporal transversality conditions, we obtain

$$
\int_{0}^{\infty} e^{-\rho t} \lambda^{*} \frac{\partial\left(x-x^{*}\right)}{\partial t} d t=\int_{0}^{\infty} e^{-\rho t}\left(\varepsilon y+\varepsilon^{2} \xi\right)\left(\dot{\lambda}^{*}-\rho \lambda^{*}\right) d t .
$$

Integrating twice by parts the term $\int_{z \in Z} \int_{0}^{\infty} e^{-\rho t} \lambda^{*} D \frac{\partial^{2}\left(x-x^{*}\right)}{\partial z^{2}} d t d z$ and using 
appropriate spatial transversality conditions, we obtain

$\int_{z \in Z} \int_{0}^{\infty} e^{-\rho t} \lambda^{*} D \frac{\partial^{2}\left(x-x^{*}\right)}{\partial z^{2}} d t d z=-\int_{z \in Z} \int_{0}^{\infty} e^{-\rho t}\left(\varepsilon y+\varepsilon^{2} \xi\right) D \frac{\partial^{2} \lambda^{*}}{\partial z^{2}} d t d z$

We substitute for the perturbed path $x=x^{*}+\varepsilon y+\varepsilon^{2} \xi+o\left(\varepsilon^{2}\right)$ into (98) and we expand the term $\mathcal{G}-\mathcal{G}^{0}$ as a Taylor series around the optimal path $\left(x^{*}(t, z), u^{*}(t, z), v^{*}(t, z), \lambda^{*}(t, z)\right)$ to obtain

$$
\mathcal{G}-\mathcal{G}^{0}=\kappa^{\prime} \nabla \mathcal{G}^{*}+\frac{1}{2} \kappa^{\prime} \nabla^{2} \mathcal{G}^{*} \kappa+\text { higher order terms }
$$

where $\nabla \mathcal{G}, \nabla^{2} \mathcal{G}$ are the first- and second-order Fréchet derivatives of the Hamiltonian function evaluated at the optimal paths. and

$$
\begin{aligned}
\kappa & =\left(\varepsilon y+\varepsilon^{2} \xi, \varepsilon \gamma, \varepsilon \zeta, \varepsilon\left(\mathbf{K}_{0} y\right)+\varepsilon^{2}\left(\mathbf{K}_{0} \xi\right), \varepsilon\left(\mathbf{K}_{0} \gamma\right), \varepsilon(\mathbf{K} y)+\varepsilon^{2}(\mathbf{K} \xi), \varepsilon(\mathbf{K} \gamma)\right)^{\prime}, \text { or } \\
\kappa & =\left(\varepsilon y+\varepsilon^{2} \xi, \varepsilon \gamma, \varepsilon \zeta, \varepsilon Y_{0}+\varepsilon^{2} Y_{0 \xi}, \varepsilon G_{0}, \varepsilon Y+\varepsilon^{2} Y_{\xi}, \varepsilon G\right)^{\prime} .
\end{aligned}
$$

Then (98) becomes

$$
\begin{aligned}
& J(u, v)-J\left(u^{*}, v^{*}\right)= \\
& \int_{z \in Z} \int_{0}^{\infty} e^{-\rho t}\left[\kappa^{\prime} \nabla \mathcal{G}^{*}+\frac{1}{2} \kappa^{\prime} \nabla^{2} \mathcal{G}^{*} \kappa+\left(\varepsilon y+\varepsilon^{2} \xi\right)\left(\dot{\lambda}^{*}-\rho \lambda^{*}+D \frac{\partial^{2} \lambda^{*}}{\partial z^{2}}\right)+\right] d t d z .
\end{aligned}
$$

The term $\kappa^{\prime} \nabla \mathcal{G}$ can be expanded as

$$
\begin{aligned}
\kappa^{\prime} \nabla \mathcal{G}= & \mathcal{G}_{x}^{*}\left(\varepsilon y+\varepsilon^{2} \xi\right)+\mathcal{G}_{u}^{*} \varepsilon \gamma+\mathcal{G}_{v}^{*} \varepsilon \zeta+\mathcal{G}_{X_{0}}^{*}\left(\varepsilon\left(\mathbf{K}_{0} y\right)+\varepsilon^{2}\left(\mathbf{K}_{0} \xi\right)\right)+\mathcal{G}_{U_{0}}^{*} \varepsilon\left(\mathbf{K}_{0} \gamma\right) \\
& +\mathcal{G}_{X}^{*}\left(\varepsilon(\mathbf{K} y)+\varepsilon^{2}(\mathbf{K} \xi)\right)+\mathcal{G}_{U}^{*} \varepsilon(\mathbf{K} \gamma) .
\end{aligned}
$$

By appropriate change in the order of integration we can write

$$
\begin{aligned}
\int_{z \in Z} \mathcal{G}_{X}^{*}\left(\varepsilon(\mathbf{K} y)+\varepsilon^{2}(\mathbf{K} \xi)\right) d z & =\int_{z \in Z}\left[\varepsilon y\left(\mathbf{K} \mathcal{G}_{X}^{*}\right)+\varepsilon^{2} \xi\left(\mathbf{K} \mathcal{G}_{X}^{*}\right)\right] d z \\
\int_{z \in Z} \mathcal{G}_{U}^{*} \varepsilon(\mathbf{K} \gamma) d z & =\int_{z \in Z} \varepsilon \gamma\left(\mathbf{K} \mathcal{G}_{U}^{*}\right) d z
\end{aligned}
$$

and the same for the corresponding $X_{0}, U_{0}$ kernels. Assume that

$$
\begin{aligned}
& \mathcal{G}_{u}^{*}+\left(\mathbf{K}_{0} \mathcal{G}_{U_{0}}^{*}\right)+\left(\mathbf{K} \mathcal{G}_{U}^{*}\right)=0, \mathcal{G}_{v}^{*}=0 \\
& \dot{\lambda}^{*}-\rho \lambda^{*}+D \frac{\partial^{2} \lambda^{*}}{\partial z^{2}}+\mathcal{G}_{x}^{*}+\left(\mathbf{K}_{0} \mathcal{G}_{X_{0}}^{*}\right)+\mathbf{K} \mathcal{G}_{X}^{*}=0 .
\end{aligned}
$$


But (106)-(107) are the optimality conditions for problem (79), then (103) becomes

$$
J(u, v)-J\left(u^{*}, v^{*}\right)=\int_{z \in Z} \int_{0}^{\infty} e^{-\rho t} \frac{1}{2} \kappa^{\prime} \nabla^{2} \mathcal{G}^{*} \kappa,
$$

where all the terms of the quadratic form $\kappa^{\prime} \nabla^{2} \mathcal{G}^{*} \kappa$ are multiples of $\varepsilon^{2}$ or higher terms. Since the approximation (108) holds along the optimal path, then assuming that state dynamics start in the neighborhood of the FOSS, dividing by $\varepsilon^{2}$ and taking the limit as $\varepsilon \rightarrow 0$, we define the approximation at the FOSS as

$$
J(u, v)-J\left(\bar{u}^{*}, \bar{v}^{*}\right)=\int_{z \in Z} \int_{0}^{\infty} e^{-\rho t} \frac{1}{2} \kappa^{\prime} \nabla^{2} \overline{\mathcal{G}}^{*} \kappa,
$$

where all derivatives are evaluated at the FOSS. Assuming that state dynamics start in the neighborhood of the FOSS, dividing by $\varepsilon^{2}$ and taking the limit as $\varepsilon \rightarrow 0$ we can obtain a 'good LQ approximation' of the nonlinear problem (79) by substituting $f_{0}\left(x, u, X_{0}, U_{0}\right)+\theta v^{2}$ by $\frac{1}{2} \kappa_{0}^{\prime} \nabla^{2} \overline{\mathcal{G}}^{*} \kappa_{0}+\theta v^{2}$ where $\kappa_{0}=\left(y, \gamma, Y_{0}, G_{0}, Y, G\right)$. Writing $\nabla^{2} \overline{\mathcal{G}}^{*}=Q$ and abusing notation by setting $\left(y, \gamma, Y_{0}, G_{0}, Y, G\right)=\left(x, u, X_{0}, U_{0}, X, U\right)=\xi^{\prime}$ and $\zeta=v$, the approximating LQ penalty robust control problem can be written as

$$
\begin{aligned}
& \max _{u(t, z)} \min _{v(t, z)} \int_{z \in Z} \int_{0}^{\infty} e^{-\rho t} \frac{1}{2}\left[\xi^{\prime} Q \xi+\theta v^{2}\right] d t d z \\
& \text { subject to } \\
& \frac{\partial x}{\partial t}=f_{x}^{*} x+f_{u}^{*} u+f_{X}^{*} X+f_{U}^{*} U+f_{v}^{*} v+D \frac{\partial^{2} x}{\partial z^{2}}, x(0, z)=x_{0}(z),
\end{aligned}
$$

which is problem (13) with $\left(f_{x}^{*}, f_{u}^{*}, f_{X}^{*}, f_{U}^{*}, f_{v}^{*}\right)=(A, B, \bar{A}, \bar{B}, C)$ and $Q=$ $\left[q_{i j}\right], i, j=1, \ldots, 6$ is a $(6 \times 6)$ symmetric matrix.

\section{Appendix 2: The derivation of the quadratic form $\hat{w}^{\prime} \hat{Q} \hat{w}$}

In the quadratic form $\hat{w}^{\prime} \hat{Q} \hat{w}$ the matrix $\hat{Q}$ is a symmetric $(2 \times 2)$ matrix $\left[\hat{q}_{i j}\right], i, j=1,2$. Its elements are derived from the quadratic form $\xi^{\prime} Q \xi$ using the convolution theorem for the Fourier transform (e.g. Bracewell $2000)$. Let $\left(x, u, \mathbf{K}_{x}^{0} x, \mathbf{K}_{u}^{0} x, \mathbf{K}_{x} x, \mathbf{K}_{u} u\right)=\xi^{\prime}=\left(\xi_{1}, \xi_{2}, \xi_{3}, \xi_{4}, \xi_{5}, \xi_{6}\right)$, where $\left(\mathbf{K}_{x}^{0} x, \mathbf{K}_{u}^{0} x, \mathbf{K}_{x} x, \mathbf{K}_{u} u\right)=\left(X_{0}, U_{0}, X, U\right)$. Then $\xi^{\prime} Q \xi=\sum_{i, j=1}^{6} q_{i j} \xi_{i} \xi_{j}$.

To make the derivation clear, take $i=1$. Then the sum of the terms of 
the quadratic form corresponding to $i=1$ is

$$
Q_{1}=q_{11} x^{2}+q_{12} x u+q_{13} x\left(\mathbf{K}_{x}^{0} x\right)+q_{14} x\left(\mathbf{K}_{u}^{0} u\right)+q_{15} x\left(\mathbf{K}_{x} x\right)+q_{16} x\left(\mathbf{K}_{u} u\right) .
$$

Using the convolution theorem for the DFT we obtain:

$$
\begin{aligned}
& \left(\mathbf{K}_{x}^{0} x\right)(t, z) \rightarrow \hat{\mathbf{K}}_{x}^{0}(\omega) \hat{x}(\omega),\left(\mathbf{K}_{u}^{0} u\right)(t, z) \rightarrow \hat{\mathbf{K}}_{u}^{0}(\omega) \hat{u}(\omega), \\
& \left(\mathbf{K}_{x} x\right)(t, z) \rightarrow \hat{\mathbf{K}}_{x}(\omega) \hat{x}(\omega),\left(\mathbf{K}_{u} u\right)(t, z) \rightarrow \hat{\mathbf{K}}_{u}(\omega) \hat{u}(\omega),
\end{aligned}
$$

then

$$
\begin{aligned}
Q_{1} \rightarrow & {\left[q_{11}+q_{13} \hat{\mathbf{K}}_{x}^{0}(\omega)+q_{15} \hat{\mathbf{K}}_{x}(\omega)\right] \hat{x}^{2}(\omega)+} \\
& {\left[q_{12}+q_{14} \hat{\mathbf{K}}_{u}^{0}(\omega)+q_{16} \hat{\mathbf{K}}_{u}(\omega)\right] \hat{x}(\omega) \hat{u}(\omega)=\hat{Q}_{1}(\omega) . }
\end{aligned}
$$

The same can be repeated for $i=2, \ldots, 6$. It is noted that the sum of the pure quadratic terms, which is

$$
q_{11} x^{2}+q_{22} u^{2}+q_{33}\left(K_{x}^{0} x\right)^{2}+q_{44} x\left(K_{u}^{0} u\right)^{2}+q_{55} x\left(K_{x} x\right)^{2}+q_{66}\left(K_{u} x\right)^{2},
$$

becomes, after using the convolution and the power theorems,

$$
\begin{aligned}
& {\left[q_{11}+q_{33}\left(\hat{\mathbf{K}}_{x}^{0}(\omega)\right)^{2}+q_{55}\left(\hat{\mathbf{K}}_{x}(\omega)\right)^{2}\right] \hat{x}^{2}(\omega)+} \\
& {\left[q_{22}+q_{44}\left(\hat{\mathbf{K}}_{u}^{0}(\omega)\right)^{2}+q_{66}\left(\hat{\mathbf{K}}_{u}(\omega)\right)^{2}\right] \hat{u}^{2}(\omega) .}
\end{aligned}
$$

After performing all calculations and taking common factors of $\hat{x}^{2}(\omega)$, $\hat{u}^{2}(\omega), \hat{x}(\omega) \hat{u}(\omega)$, we obtain the quadratic form $\hat{w}^{\prime} \hat{Q} \hat{w}$ where the elements of matrix $\hat{Q}(\omega)$ are defined in the dual space $\hat{Z}$, or the frequency domain, and depend on the spatial kernels.

Appendix 3: The parameters of the value function of the commercial fishery

The parameters of the value function are determined by the solution of 
the following system:

$$
\begin{aligned}
& -P_{2}+A_{2} P_{3}-\frac{1}{2} \hat{q}_{22}+\frac{2 B_{2}^{2} P_{2}^{2}}{\hat{q}_{33}}+\frac{2 A_{3} B_{2} P_{2} P_{3}}{\hat{q}_{33}}+\frac{1}{2} \frac{A_{3}^{2} P_{3}^{2}}{\hat{q}_{33}}- \\
& \frac{2 B_{2} P_{2} \hat{q}_{23}}{\hat{q}_{33}}-\frac{A_{3} P_{3} \hat{q}_{23}}{\hat{q}_{33}}+\frac{1}{2} \frac{\hat{q}_{23}^{2}}{\hat{q}_{33}}+\frac{1}{2} \frac{C^{2} P_{3}^{2}}{\theta}=0 \\
& 2 A_{2} P_{1}+2 B_{1} P_{2}-P_{3}+A_{1} P_{3}-\hat{q}_{21}+\frac{4 A_{3} B_{2} P_{1} P_{2}}{\hat{q}_{33}}+\frac{2 A_{3}^{2} P_{1} P_{3}}{\hat{q}_{33}}+ \\
& \frac{2 B_{2}^{2} P_{2} P_{3}}{\hat{q}_{33}}+\frac{A_{3} B_{2} P_{3}^{2}}{\hat{q}_{33}}-\frac{2 A_{3} P_{1} \hat{q}_{23}}{\hat{q}_{33}}-\frac{B_{2} P_{3} \hat{q}_{23}}{\hat{q}_{33}}-\frac{2 B_{2} P_{2} \hat{q}_{31}}{\hat{q}_{33}}- \\
& \frac{A_{3} P_{3} \hat{q}_{31}}{\hat{q}_{33}}+\frac{\hat{q}_{23} \hat{q}_{31}}{\hat{q}_{33}}+\frac{2 C^{2} P_{1} P_{3}}{\theta}=0 \\
& -P_{1}+2 A_{1} P_{1}+B_{1} P_{3}-\frac{1}{2} \hat{q}_{11}+\frac{2 A_{3}^{2} P_{1}^{2}}{\hat{q}_{33}}+\frac{2 A_{3} B_{2} P_{1} P_{3}}{\hat{q}_{33}}+\frac{1}{2} \frac{B_{2}^{2} P_{3}^{2}}{\hat{q}_{33}}- \\
& \frac{2 A_{3} P_{1} \hat{q}_{31}}{\hat{q}_{33}}-\frac{B_{2} P_{3} \hat{q}_{31}}{\hat{q}_{33}}+\frac{1}{2} \frac{\hat{q}_{31}^{2}}{\hat{q}_{33}}+\frac{2 C^{2} P_{1}^{2}}{\theta}=0 .
\end{aligned}
$$

\section{References}

Athans, M. and P. Falb (1966). Optimal Control. New York: McGrawHill.

Bamieh, B., F. Paganini, and M. Dahleh (2002). Distributed control of spatially invariant systems. IEEE Transactions on automatic control $47(7)$, 1091-1107.

Benigno, P. and M. Woodford (2006). Linear-quadratic approximation of optimal policy problems. NBER Working Paper No. 12672.

Boucekkine, R., C. Camacho, and B. Zou (2009). Bridging the gap between growth theory and the new economic geography: the spatial Ramsey model. Macroeconomic Dynamics 13, 20-45.

Bracewell, R.N. (2000), The Fourier Transform and its Applications, 3rd Edition, Boston: McGraw-Hill.

Brock, W. and A. Malliaris (1989). Differential Equations, Stability and Chaos in Dynamic Economics. Amsterdam: North-Holland.

Brock, W. and A. Xepapadeas (2008). Diffusion-induced instability and pattern formation in infinite horizon recursive optimal control. Journal of Economic Dynamics and Control 32, 2745-2787.

Brock, W. and A. Xepapadeas (2009). The emergence of optimal agglomeration in dynamic economics. University of Wisconsin Working Papers, http://www.ssc.wisc.edu/ ${ }^{\sim}$ wbrock/Brock_Xepapadeas_Optimal_Agglomeration.pdf 
Brock, W. and A. Xepapadeas (2010). Pattern formation, spatial externalities and regulation in coupled economic-ecological systems. Journal of Environmental Economics and Management 59, 149-164.

Chu, E. (2008). Discrete and Continuous Fourier Transforms - Analysis, Applications and Fast Algorithms. Boca-Raton: Chapman \& Hall/CRC.

Curtain, R., O. Iftime, and H. Zwart (2008). System theoretic properties of platton type systems. Proceedings of 4 7th IEEE Conference on Decision and Control, 1442-1447.

Desmet, K. and E. Rossi-Hansberg (2009). Spatial development. Working Paper, Universidad Carlos III and Princeton University.

Gilboa, I. and D. Schmeidler (1989). Maxmin expected utility with nonunique prior. Journal of Mathematical Economics 18, 141-153.

Haldane, A. (2009). Rethinking the Financial Network. Speech delivered at the Financial Students Association, Amsterdam, Bank of England, http://www.bankofengland.co.uk/publications/speeches/2009/speech386.pdf.

Hansen, L. and T. Sargent (2001). Robust control and model uncertainty. American Economic Review 91, 60-66.

Hansen, L., T. Sargent, G. Turmuhambetova, and N. Williams (2006). Robust control and model misspecification. Journal of Economic Theory 128, 45-90.

Hansen, L. and T. Sargent (2008). Robustness. Princeton: Princeton University Press.

JET (2006). Symposium on model uncertainty and robustness. Journal of Economic Theory 128, 1-163.

Krugman, P. (1996). The Self-Organizing Economy. Oxford: Blackwell.

Leizarowitz, A. (2008). Turnpike properties of a class of aquifer control problems. Automatica 44, 1460-1470.

Magill, M. (1977a). Some new results on the local stability of the process of capital accumulation. Journal of Economic Theory 15, 174-10.

Magill, M. (1977b). A local analysis of N-sector capital accumulation under uncertainty. Journal of Economic Theory 15, 211-219.

Murray, J. (2003). Mathematical Biology, Volumes I and II, 3rd Edn. Berlin: Springer.

Rudin, W. (1962). Fourier Analysis on Groups. New York: InterscienceWiley.

Salmon, M. (Ed.) (2002). Special Issue on Robust and Risk Sensitive 
Decision Theory. Macroeconomic Dynamics 6(1).

Sanchirico, J. and J. Wilen (1999). Bioeconomics of spatial exploitation in a patchy environment. Journal of Environmental Economics and Management 37(2), 129-150.

Smith, M., J. Sanchirico, and J. Wilen (2009). The economics of spatialdynamic processes: applications to renewable resources. Journal of Environmental Economics and Management 57, 104-121.

Smith, V. (1969). On models of commercial fishing. Journal of Political Economy 77, 181-198.

Turing, A. (1952). The chemical basis of morphogenesis. Philosophical Transactions of the Royal Society of London 237, 37-72. 